\title{
OPTIMAL ERROR ESTIMATES FOR FEM APPROXIMATIONS OF DYNAMIC NONLINEAR SHALLOW SHELLS*
}

\author{
IRENA LASIECKA ${ }^{1}$ AND RICH MARCHAND ${ }^{2}$
}

\begin{abstract}
Finite element semidiscrete approximations on nonlinear dynamic shallow shell models in considered. It is shown that the algorithm leads to global, optimal rates of convergence. The result presented in the paper improves upon the existing literature where the rates of convergence were derived for small initial data only [19].
\end{abstract}

Mathematics Subject Classification. 65, 35.

Received: July 20, 1998. Revised: May 3, 1999.

\section{INTRODUCTION}

The main goal of this paper is to derive optimal rates of convergence, i.e. reconstructing the best approximation properties of the underlying finite dimensional subspaces, for semidiscrete finite element (FEM) approximations of a nonlinear dynamic shallow shell model. In contrast with the literature [19], our results do not require any assumptions on the "smallness" of the initial data.

\subsection{The model}

The model to be considered is a nonlinear model for a thin shallow dynamic shell $[1,7,13,19]$. We begin by describing the notation to be used. Throughout this paper, the summation convention will be used with Greek letters belonging to the set $\{1,2\}$ and Latin letters belonging to the set $\{1,2,3\}$. The middle surface $S$ of the shell is defined to be the image of a connected bounded open set $\Omega \subset \mathcal{E}^{2}$ with boundary $\Gamma$ under the mapping $\Psi:\left(\xi^{1}, \xi^{2}\right) \in \bar{\Omega} \rightarrow \mathcal{E}^{3}$ where $\Psi \in\left[C^{3}(\bar{\Omega})\right]^{3}$ and $\mathcal{E}^{n}$ is the $n$-dimensional Euclidean Space. Then for any point on the surface of the shell, it is assumed that the two tangent vectors given by $\mathbf{a}_{\alpha}=\partial \Psi / \partial \xi^{\alpha}$ are linearly independent. Moreover, these two vectors along with the normal vector, $\mathbf{a}_{3}=\mathbf{a}_{1} \times \mathbf{a}_{2} /\left|\mathbf{a}_{1} \times \mathbf{a}_{2}\right|$ define a covariant basis for a local reference frame on the surface of the shell. Hereafter, the notation $\Phi,{ }_{\alpha}=\partial \Phi / \partial \xi^{\alpha}$ for any point $\left(\xi^{1}, \xi^{2}\right) \in \bar{\Omega}$ will be used.

The contravariant basis for the tangent plane at any point on the surface of the shell is given by the two vectors $\mathbf{a}^{\beta}$ defined by the relation $\mathbf{a}_{\alpha} \cdot \mathbf{a}^{\beta}=\delta_{\alpha}^{\beta}$. The contravariant and covariant vectors are associated by

\footnotetext{
Keywords and phrases. Finite elements, nonlinear dynamic shells, optimal error estimates, global existence and uniqueness.

* I. Lasiecka was partially supported by National Science Foundation Grant NSF DMS-98-04056 and Army Research Office Grant ARO DAAH04-96-1-0059. The material of Rich Marchand was partially supported by a National Research Council Postdoctoral Research Fellowship.

1 Department of Mathematics, University of Virginia, Charlottesville, VA 22903, USA.

2 Department of Mathematics Sciences, United States Military Academy, West Point, NY 10996, USA.
} 
the well-known relationships

$$
\mathbf{a}_{\alpha}=a_{\alpha \beta} \mathbf{a}^{\beta}, \quad \mathbf{a}^{\alpha}=a^{\alpha \beta} \mathbf{a}_{\beta}, \quad a_{\alpha \beta}=\mathbf{a}_{\alpha} \cdot \mathbf{a}_{\beta}=a_{\beta \alpha}, \quad \text { and } \quad a^{\alpha \beta}=\mathbf{a}^{\alpha} \cdot \mathbf{a}^{\beta}=a^{\beta \alpha},
$$

where the matrix $\left(a_{\alpha \beta}\right)$ represents the first fundamental form of the surface with its inverse given by the matrix $\left(a^{\alpha \beta}\right)$.

The second fundamental form, denoted by $\left(b_{\alpha \beta}\right)$, measures the normal curvatures of the middle surface of the shell. It is defined by

$$
b_{\alpha \beta}=b_{\beta \alpha}=-\mathbf{a}_{\alpha} \cdot \mathbf{a}_{3, \beta}=\mathbf{a}_{3} \cdot \mathbf{a}_{\alpha, \beta} .
$$

The Christoffel symbols given by $\Gamma_{\beta \lambda}^{\alpha}=\mathbf{a}^{\alpha} \cdot \mathbf{a}_{\beta, \lambda}$ give rise to the following covariant derivatives for the displacement vector of the middle surface $\mathbf{u}\left(\xi^{1}, \xi^{2}\right)=u_{i} \mathbf{a}^{i}$ in a fixed reference frame:

$$
u_{\alpha \mid \beta}=u_{\alpha, \beta}-\Gamma_{\alpha \beta}^{\lambda} u_{\lambda} \text { and } u_{3 \mid \alpha \beta}=u_{3, \alpha \beta}-\Gamma_{\alpha \beta}^{\lambda} u_{3, \lambda} .
$$

For a more complete description of the geometrical considerations and defining characteristics of thin shallow shells, see $[5,13]$. For more information on tensor analysis, see $[8,12]$.

We represent the vector function for all three displacements by $\mathbf{u}=\left(u_{1}, u_{2}, u_{3}\right)$, while in-surface displacements are given by $\vec{u}=\left(u_{1}, u_{2}\right)$ and the transverse displacement is denoted by $u_{3}$. In addition, the linear strain tensor is denoted by $\epsilon_{\alpha \beta}(\vec{u})=\frac{1}{2}\left(u_{\alpha \mid \beta}+u_{\beta \mid \alpha}\right)$. Then from Koiter's development of nonlinear shallow shell theory in [13], the middle surface strain tensor $\gamma_{\alpha \beta}$ and the change of curvature tensor $\rho_{\alpha \beta}$ are given respectively by

$$
\gamma_{\alpha \beta}(\mathbf{u})=\epsilon_{\alpha \beta}(\vec{u})-b_{\alpha \beta} u_{3}+\frac{1}{2} u_{3, \alpha} u_{3, \beta} \text { and } \rho_{\alpha \beta}(\mathbf{u})=u_{3 \mid \alpha \beta} .
$$

These strain measures, along with an appropriate constitutive law, are used to derive corresponding stress measures. The exact form of the stress measures depends on the shell material. Thus, we assume the simplest possible scenario as done in [2] which is based on the assumptions that the shell consists of an elastic, homogeneous, and isotropic material and the strains are small everywhere. Additionally, we assume that all nonzero stress components are imposed on surfaces which are parallel to the middle surface of the shell. Then the stress resultants and moments respectively take on the forms

$$
n^{\alpha \beta}(\mathbf{u})=e E^{\alpha \beta \lambda \mu} \gamma_{\lambda \mu}(\mathbf{u}) \text { and } m^{\alpha \beta}(\mathbf{u})=\frac{e^{3}}{12} E^{\alpha \beta \lambda \mu} \rho_{\lambda \mu}(\mathbf{u}),
$$

where $e$ represents the thickness of the shell, and the tensor of elastic moduli is given by

$$
E^{\alpha \beta \lambda \mu}=\frac{E}{2(1+\nu)}\left[a^{\alpha \lambda} a^{\beta \mu}+a^{\alpha \mu} a^{\beta \lambda}+\frac{2 \nu}{1-\nu} a^{\alpha \beta} a^{\lambda \mu}\right] .
$$

Here, $E$ is Young's modulus and $\nu$ is Poisson's ratio for the material. See [4] for a proof of the positivity of the tensor of elastic moduli. Let $\vec{p} \in\left[L_{1}((0, T) \times \Omega)\right]^{2}, q \in L_{1}\left((0, T) ; H^{-1}(\Omega)\right)$ denote the prescribed body forces and $\rho>0$ denotes mass density. Additionally, we let $\dot{u}$ represent differentiation with respect to time and make use of the following notation

$$
(u, v)=\int_{\Omega} u v \sqrt{a} \mathrm{~d} \xi_{1} \mathrm{~d} \xi_{2} ;|u|_{s, \Omega} \equiv|u|_{H^{s}(\Omega)}, \quad|u|_{s, p} \equiv|u|_{L_{p}\left(0, T ; H^{s}(\Omega)\right)} \text { for } 1 \leq p \leq \infty
$$

where $a=\operatorname{det}\left(a_{\alpha \beta}\right)>0$.

$$
|(\vec{p}, q)| \equiv \int_{0}^{T}\left[|\vec{p}|_{0, \Omega}+|q|_{-1, \Omega}\right] \mathrm{d} t
$$


The model to be considered is given in the following variational form in which we look for solutions $\mathbf{u}=$ $\left(u_{1}, u_{2}, u_{3}\right)=\left(\vec{u}, u_{3}\right) \in\left[H_{0}^{1}(\Omega)\right]^{2} \cap H_{0}^{2}(\Omega)$ such that

$$
\begin{array}{r}
\left(\rho e a^{\alpha \beta} \ddot{u}_{\beta}, v_{\alpha}\right)+\left(e E^{\alpha \beta \lambda \mu} \gamma_{\alpha \beta}(\mathbf{u}), \epsilon_{\lambda, \mu}(\vec{v})\right)=(\vec{p}, \vec{v}) \\
\left(\rho e \ddot{u}_{3}, v_{3}\right)+\gamma\left(\rho e \ddot{u}_{3, \alpha}, v_{3, \alpha}\right)+\left(\frac{e^{3}}{12} E^{\alpha \beta \lambda \mu} u_{3 \mid \alpha \beta}, v_{3 \mid \lambda \mu}\right)+\left(e E^{\alpha \beta \lambda \mu} \gamma_{\alpha \beta}(\mathbf{u}), u_{3, \lambda} v_{3, \mu}-b_{\lambda \mu} v_{3}\right)=\left(q, v_{3}\right)
\end{array}
$$

for all $\mathbf{v}=\left(v_{1}, v_{2}, v_{3}\right)=\left(\vec{v}, v_{3}\right) \in\left[H_{0}^{1}(\Omega)\right]^{2} \cap H_{0}^{2}(\Omega)$. The static version of this model is given in [5]. Our model takes into account additional considerations which include the dynamics of the shell (see [3,19, 22]. The constant $\gamma$ is proportional to the square of the thickness of the shell and is therefore assumed to be small. Some models (e.g [19]) consider $\gamma=0$. Physically, the term $\left.\gamma \ddot{u}_{3}\right|_{\alpha} ^{\alpha}$ represents rotational forces.

We also introduce the "energy" functional corresponding to the system in $(1.2,1.3)$ as

$$
E_{\gamma}(t)=E_{p}(t)+E_{k}^{\gamma}(t)
$$

where

$$
\begin{aligned}
& E_{k}^{\gamma}(t)=\frac{1}{2}\left\{\left(\rho e a^{\alpha \beta} \dot{u}_{\beta}, \dot{u}_{\alpha}\right)+\left(\rho e \dot{u}_{3}, \dot{u}_{3}\right)+\gamma\left(\rho e \dot{u}_{3, \alpha}, \dot{u}_{3, \alpha}\right)\right\} \\
& E_{p}(t)=\frac{1}{2}\left\{\left(e E^{\alpha \beta \lambda \mu} \gamma_{\alpha \beta}(\mathbf{u}), \gamma_{\lambda \mu}(\mathbf{u})\right)+\left(\frac{e^{3}}{12} E^{\alpha \beta \lambda \mu} u_{3 \mid \alpha \beta}, u_{3 \mid \lambda \mu}\right)\right\}
\end{aligned}
$$

represent the kinetic and potential energies respectively. By formal computations, one can verify that the energy of the system is bounded in terms of the initial data, i.e., $E_{\gamma}(t) \leq C\left[E_{\gamma}(0)+|(\vec{p}, q)|^{2}\right] ; 0 \leq t \leq T$. It is known that the potential energy, $E_{p}(t)$ is topologically equivalent to the norms in $\left[H^{1}(\Omega)\right]^{2} \times H^{2}(\Omega)$, so there is an a priori bound in this space. Moreover, since the nonlinear terms (cubic nonlinearities) are weakly continuous with respect to $H^{2}(\Omega)$ topology, standard Faedo-Galerkin method [17] yields the existence of weak (finite energy) solutions. A much more difficult task is to obtain uniqueness of weak solutions and additional regularity of solutions corresponding to more regular data. Indeed, the mathematical difficulty is due to dynamic nature of the problem and the fact that the nonlinear terms in the system $(1.2,1.3)$, are not locally Lipschitz, with respect to the norm induced by the energy. This makes the analysis difficult, and standard arguments used for showing well-posedness of solutions are no longer applicable as acknowledged on p. 204 of [1]. This is particularly true with respect to the uniqueness of weak (finite energy) solutions which has been an outstanding open problem even in the case of plate theory $[14,17]$. In fact, the full well-posedness/regularity theory for the model given in $(1.2,1.3)$ has been developed only very recently, and the theorem given below summarizes the main results.

Theorem 1.1. With reference to the system in (1.2, 1.3) with $\gamma>0$,

1. (Weak solutions). Let $\Omega$ be convex or $\Gamma$ sufficiently smooth $\left(C^{1}\right)$. Then for any initial data

$$
\begin{array}{ll}
\vec{u}(0, \cdot) \in\left[H_{0}^{1}(\Omega)\right]^{2}, & u_{3}(0, \cdot) \in H_{0}^{2}(\Omega), \\
\dot{\vec{u}}(0, \cdot) \in\left[L_{2}(\Omega)\right]^{2}, & \dot{u}_{3}(0, \cdot) \in H_{0}^{1}(\Omega),
\end{array}
$$

and the body forces

$$
\vec{p} \in\left[L_{1}\left((0, T) ; L_{2}(\Omega)\right)\right]^{2}, \quad q \in L_{1}\left((0, T) ; H^{-1}(\Omega)\right),
$$

there exists a unique solution

$$
\begin{gathered}
\vec{u} \in C\left([0, T] ;\left[H_{0}^{1}(\Omega)\right]^{2}\right) \cap C^{1}\left([0, T] ;\left[L_{2}(\Omega)\right]^{2}\right), \\
u_{3} \in C\left([0, T] ; H_{0}^{2}(\Omega)\right) \cap C^{1}\left([0, T] ; H_{0}^{1}(\Omega)\right) .
\end{gathered}
$$


2. (Regular solutions). Let $\Gamma$ be sufficiently smooth $\left(C^{2}\right)$. Then for any initial data and body forces such that

$$
\begin{gathered}
\vec{u}(0, \cdot) \in\left[H_{0}^{1}(\Omega)\right]^{2} \cap\left[H^{2}(\Omega)\right]^{2}, \quad u_{3}(0, \cdot) \in H_{0}^{2}(\Omega) \cap H^{3}(\Omega), \\
\dot{\vec{u}}(0, \cdot) \in\left[H_{0}^{1}(\Omega)\right]^{2}, \quad \dot{u}_{3}(0, \cdot) \in H_{0}^{2}(\Omega), \\
\vec{p} \in\left[H^{1}\left((0, T) ; L_{2}(\Omega)\right)\right]^{2}, \quad q \in H^{1}\left((0, T) ; H^{-1}(\Omega)\right),
\end{gathered}
$$

there exists a unique solution

$$
\begin{gathered}
\vec{u} \in C\left([0, T] ;\left[H^{2}(\Omega)\right]^{2}\right) \cap C^{1}\left([0, T] ;\left[H_{0}^{1}(\Omega)\right]^{2}\right), \\
u_{3} \in C\left([0, T] ; H^{3}(\Omega)\right) \cap C^{1}\left([0, T] ; H_{0}^{2}(\Omega)\right) .
\end{gathered}
$$

As already mentioned, the existence of weak solutions is standard and follows from a classical Galerkin-Faedo argument applicable to von Karman types of problems, see [17]. The more subtle issue is the uniqueness of weak solutions, stated in part (1) of the Theorem, and the regularity of solutions, stated in part (2) of the Theorem.

Indeed, the second part of Theorem 1.1 (Regular solutions) was proved in [20] (see also [16]) and relies critically on strong a priori estimates obtained for higher (than the energy) norms of solutions. The most difficult part is the uniqueness of weak solutions, which has been recently proven in [15] (see also [16] where intermediate regularity of solutions has been studied) by adapting the method used in [24].

If the rotational forces are neglected, i.e. $\gamma=0$, then the result of Theorem 1.1 is still valid, see [24], with the following differences: in part (1) the velocity component of $u_{3}$ is in $L_{2}(\Omega)$ and $q \in L_{1}\left((0, T) ; L_{2}(\Omega)\right)$, and in part (2) the displacement $u_{3}$ is in $H^{4}(\Omega)$ and $q \in H^{1}\left((0, T) ; L_{2}(\Omega)\right)$.

Remark 1.1. By iterating the same technique as used for the proof of part 2 of Theorem 1.1, one can show that by assuming more regularity on the initial data more regular solutions are obtained.

\subsection{Semidiscrete approximation}

Let $V^{h} \subset\left[H_{0}^{1}(\Omega)\right]^{2}$ and $W^{h} \subset H_{0}^{2}(\Omega)$ be finite element approximations (say piecewise linear functions in $V_{h}$ and B-splines or HET elements in $W_{h}$ ) defined on a quasi-uniform mesh, satisfying the following standard requirements (as in $[6,25])$ :

$$
\begin{gathered}
\inf _{\vec{u}^{h} \in V^{h}}\left|\vec{u}-\vec{u}^{h}\right|_{l, \Omega} \leq C h^{s-l}|\vec{u}|_{s, \Omega}, \text { for } 0 \leq s \leq S, \quad s \geq l \text { and } 0 \leq l \leq 1 \\
\inf _{u_{3}^{h} \in W^{h}}\left|u_{3}-u_{3}^{h}\right|_{l, \Omega} \leq C h^{r-l}\left|u_{3}\right|_{r, \Omega}, \text { for } 0 \leq r \leq R, \quad r \geq l \text { and } 0 \leq l \leq 2
\end{gathered}
$$

where $S$ and $R$ are the orders of the approximations. Typically, we have $S=k+1$ (resp. $R=t+1$ ), where $k$ (resp. $t$ ) stands for the order of the approximating polynomials (see Theorem 3.1.5 in [6]).

We consider the following semidiscrete approximation of the original model. Find $\mathbf{u}^{h}(t) \in V^{h} \times W^{h}$ such that

$$
\begin{gathered}
\left(\rho e a^{\alpha \beta} \ddot{u}_{\beta}^{h}, v_{\alpha}^{h}\right)+\left(e E^{\alpha \beta \lambda \mu} \gamma_{\alpha \beta}\left(\mathbf{u}^{h}\right), \epsilon_{\lambda \mu}\left(\vec{v}^{h}\right)\right)=\left(\vec{p}, \vec{v}^{h}\right) \\
\left(\rho e \ddot{u}_{3}^{h}, v_{3}^{h}\right)+\gamma\left(\rho e \ddot{u}_{3, \alpha}^{h}, v_{3, \alpha}^{h}\right)+\left(\frac{e^{3}}{12} E^{\alpha \beta \lambda \mu} u_{3 \mid \alpha \beta}^{h}, v_{3 \mid \lambda \mu}^{h}\right)+\left(e E^{\alpha \beta \lambda \mu} \gamma_{\alpha \beta}\left(\mathbf{u}^{h}\right), u_{3, \lambda}^{h} v_{3, \mu}^{h}-b_{\lambda \mu} v_{3}^{h}\right)=\left(q, v_{3}^{h}\right)
\end{gathered}
$$

for all $\mathbf{v}^{h} \in V^{h} \times W^{h}$ and $\mathbf{u}^{h}(0)=\mathbf{u}_{0}^{h} \in V^{h} \times W^{h}$.

Since the semidiscrete problem, $(1.5,1.6)$ is a system of nonlinear ODE's with locally Lipschitz (polynomial) nonlinearities, we obtain local (in time) existence and uniqueness of the solution $\mathbf{u}^{h} \in W^{2,1}\left(\left(0, T_{0}\right) ; V^{h} \times W^{h}\right)$. 
If the body forces $\vec{p}, q$ are more regular in time, say $H^{k}(0, T)$, then the corresponding solutions $\mathbf{u}^{h}$ display more time regularity, say $H^{k+2}(0, T)$. The existence of global solution follows from the a priori bound for the discrete energy function, which is stated in the next section. In fact, this argument is the same as the one used for proving the existence of weak solutions by means of Faedo-Galerkin method.

\subsection{Main results}

The main goal of this paper is to establish the rates of convergence for the semidiscrete approximation introduced above. In comparison with the literature, we note that most of the results available provide numerical analysis for the static model, see [1] and references therein. The dynamic model is much more involved due to the "hyperbolicity" of associated dynamics and, in particular, due to the unboundedness of the nonlinear terms in the energy norm (this is not the case for the static model). The paper which deals with the dynamic case $(\gamma=0)$ is [19] in which the rates of convergence are derived (see Th. 1.2) for initial data assumed to be sufficiently small. The primary aims of this work are: (i) to remove the above mentioned smallness assumption, (ii) to provide the explicit convergence estimates for the fully hyperbolic model acounting for rotational forces $(\gamma>0)$ which are uniform with respect to the value of the parameter $\gamma$. This way we also obtain the estimates for the critical case $\gamma=0$. In these regards, the main result of our paper is given in the following theorem.

Theorem 1.2. Let $\mathbf{u}$ be a sufficiently regular solution to (1.2, 1.3) and $\mathbf{u}^{h}$ be a solution to (1.5, 1.6) with $\gamma>0$. The required regularity of $\mathbf{u}$ is dictated by the norms appearing on the right-hand side of the estimate below. Assume that $\mathbf{u}_{0}^{h}$ is an approximation of the initial data $\mathbf{u}_{0}$ complying with the approximation properties in (1.4). Then the following error estimate holds for $0 \leq t \leq T$ and $2 \leq r \leq R ; 1 \leq s \leq S$, and all $\epsilon>0$ :

$$
\begin{aligned}
\left|\left(u_{\alpha}-u_{\alpha}^{h}\right)(t)\right|_{1, \Omega} & +\left|\left(\dot{u}_{\alpha}-\dot{u}_{\alpha}^{h}\right)(t)\right|_{0, \Omega}+\left|\left(u_{3}-u_{3}^{h}\right)(t)\right|_{2, \Omega}+\left|\left(\dot{u}_{3}-\dot{u}_{3}^{h}\right)(t)\right|_{0, \Omega}+\gamma\left|\left(\dot{u}_{3}-\dot{u}_{3}^{h}\right)(t)\right|_{1, \Omega} \\
\leq & \left(h^{(s-1)}+h^{(r-2)}\right) C_{T . \epsilon}\left(E_{\gamma}(0),|(\vec{p}, q)|,\left|\dot{u}_{3}\right|_{1+\epsilon, \infty},\left|\dot{u}_{\alpha}\right|_{\epsilon, \infty}\right)\left[\left|u_{\alpha}\right|_{s, 2}+\left|\dot{u}_{\alpha}\right|_{s, 2}+\left|\ddot{u}_{\alpha}\right|_{s-1,2}\right. \\
& \left.+\left|u_{3}\right|_{r, 2}+\left|\dot{u}_{3}\right|_{r, 2}+\left|\ddot{u}_{3}\right|_{r-2,2}+\gamma\left|\ddot{u}_{3}\right|_{r-1,2}\right]
\end{aligned}
$$

where the function $C_{T, \epsilon}$ is continuous in its arguments, independent of $h>0$ and independent of $0 \leq \gamma<M$.

Recall that we are making use of the notation $|u|_{s, p} \equiv|u|_{L_{p}\left(0, T ; H^{s}(\Omega)\right)}$. In the special case when $\gamma=0$, we obtain the following corollary.

Corollary 1.1. Case: $\gamma=0$.

Let $\mathbf{u}$ be a sufficiently regular solution to $(1.2,1.3)$ and $\mathbf{u}^{h}$ be a solution to $(1.5,1.6)$ with $\gamma=0$, then the following error estimate holds:

$$
\begin{aligned}
\left|\left(u_{\alpha}-u_{\alpha}^{h}\right)(t)\right|_{1, \Omega} & +\left|\left(\dot{u}_{\alpha}-\dot{u}_{\alpha}^{h}\right)(t)\right|_{0, \Omega}+\left|\left(u_{3}-u_{3}^{h}\right)(t)\right|_{2, \Omega}+\left|\left(\dot{u}_{3}-\dot{u}_{3}^{h}\right)(t)\right|_{0, \Omega} \\
\leq & \left(h^{(s-1)}+h^{(r-2)}\right) C_{T, \epsilon}\left(E(0),|(\vec{p}, q)|,\left|\dot{u}_{3}\right|_{1+\epsilon, \infty},\left|\dot{u}_{\alpha}\right|_{\epsilon, \infty}\right)\left[\left|u_{\alpha}\right|_{s, 2}+\left|\dot{u}_{\alpha}\right|_{s, 2}+\left|\ddot{u}_{\alpha}\right|_{s-1,2}\right. \\
& \left.+\left|u_{3}\right|_{r, 2}+\left|\dot{u}_{3}\right|_{r, 2}+\left|\ddot{u}_{3}\right|_{r-2,2}\right]
\end{aligned}
$$

Remark 1.2. In the special case of linear splines used to approximate $\vec{u}_{\alpha}$, and B-splines, used to approximate the vertical displacement $u_{3}$ (so $\left.S=2, R=3\right)$, the decay rates given in Theorem 1.2 provide the estimate

$$
\begin{aligned}
\left|\left(u_{\alpha}-u_{\alpha}^{h}\right)(t)\right|_{1, \Omega} & +\left|\left(\dot{u}_{\alpha}-\dot{u}_{\alpha}^{h}\right)(t)\right|_{0, \Omega}+\left|\left(u_{3}-u_{3}^{h}\right)(t)\right|_{2, \Omega}+\left|\left(\dot{u}_{3}-\dot{u}_{3}^{h}\right)(t)\right|_{0, \Omega}+\gamma\left|\left(\dot{u}_{3}-\dot{u}_{3}^{h}\right)(t)\right|_{1, \Omega} \\
\leq & h C_{T, \epsilon}\left(E_{\gamma}(0),|(\vec{p}, q)|,\left|\dot{u}_{3}\right|_{1+\epsilon, \infty},\left|\dot{u}_{\alpha}\right|_{\epsilon, \infty}\right)\left[\left|u_{\alpha}\right|_{2,2}+\left|\dot{u}_{\alpha}\right|_{2,2}+\left|\ddot{u}_{\alpha}\right|_{1,2}\right. \\
& \left.\quad+\left|u_{3}\right|_{3,2}+\left|\dot{u}_{3}\right|_{3,2}+\left|\ddot{u}_{3}\right|_{1,2}+\gamma\left|\ddot{u}_{3}\right|_{2,2}\right]
\end{aligned}
$$

where the function $C_{T, \epsilon}$ is continuous in its arguments, independent of $h>0$ and independent of $0 \leq \gamma<M$. 
The error estimates obtained are optimal, in the sense that they reconstruct the best approximation properties of the underlying finite dimensional subspaces, and global, in the sense that there is no need to restrict the size of the initial data. We repeat that the main mathematical novelty and difficulty of the problem is due to the unbounded nature of the nonlinear terms with respect to the topology induced by the energy function. This was the reason for the "smallness" assumption in [19], where the result of Corollary 1.1 was established under the assumption that the energy norms of the initial data are sufficiently small. In our case, we are able to dispense with the above restriction by applying tools of nonlinear interpolation theory.

Remark 1.3. By taking advantage of special "elliptic/static projections" (see [1]: p.190) one could obtain superconvergence of the estimates in Theorem 1.2 by considering the error expressed in terms of $\mathbf{u}^{h}(t)$ and these projections. In fact, in this case we can obtain the error $\mathcal{O}\left(h^{s}+h^{r-1}\right)$.

Remark 1.4. The same results as those stated in Theorem 1.2 can be applied to the shell model where the dynamic terms are modeled by the form (see [3]: p. 258, formula (1.2))

$$
\begin{aligned}
b(\ddot{\mathbf{u}}, \mathbf{v})= & \int_{\Omega} \rho e\left\{\left[1+\gamma\left(b_{1}^{1} b_{2}^{2}-b_{1}^{2} b_{2}^{1}\right)\right]\left[a^{\alpha \beta} \ddot{u}_{\alpha} v_{\beta}+\ddot{u}_{3} v_{3}\right]+\gamma^{\alpha \beta}\left[\left(\ddot{u}_{3 \mid \alpha}+b_{\alpha}^{\lambda} \ddot{u}_{\lambda}\right)\left(v_{3 \mid \beta}+b_{\beta}^{\mu} v_{\mu}\right)\right.\right. \\
& \left.\left.+\left(\ddot{u}_{\alpha} v_{3 \mid \beta}+\ddot{u}_{3 \mid \alpha} v_{\beta}+2 b_{\alpha}^{\lambda} \ddot{u}_{\lambda} v_{\beta}\right) b_{\eta}^{\eta}\right]\right\} \sqrt{a} \mathrm{~d} \xi^{1} \mathrm{~d} \xi^{2}
\end{aligned}
$$

where $\gamma=e^{2} / 12$. However, since the arguments are conceptually the same (due to the boundedness and coercivity, with respect to energy norm, of the bilinear form $b(\ddot{\mathbf{u}}, \mathbf{v}))$, we opted for a simpler form of the model.

Remark 1.5. Note that the algorithm considered in this paper accounts for the approximation of displacements/velocities only. In particular, it does not account for approximations of the geometry or integration. However, by using the results/methods of [1] this can be integrated with the analysis presented in this paper. Also, the analysis presented here could be extended to other FEM approximations such as Mixed Methods which are very popular and efficient for plates/shells problems, see [1,9-11].

The remainder of this paper is devoted to the proof of the Theorem 1.2.

\section{StABILITY AND ERROR EQUATION}

The following symmetries will be used frequently.

$$
\left\{\begin{array}{l}
b_{\alpha \beta}=b_{\beta \alpha} \\
a_{\alpha \beta}=a_{\beta \alpha}, \quad a_{\alpha \beta} u_{\alpha} u_{\beta}>0 \\
E^{\alpha \beta \lambda \mu}=E^{\lambda \mu \alpha \beta}=E^{\alpha \beta \mu \lambda}
\end{array}\right.
$$

In addition, the next four inequalities are taken from [1]:

$$
E^{\alpha \beta \lambda \mu} \gamma_{\alpha \beta} \gamma_{\lambda \mu} \geq C \sum_{\alpha, \beta=1}^{2}\left|\gamma_{\alpha \beta}\right|^{2}
$$

for any tensor $\gamma_{\alpha \beta}$, uniformly in $\bar{\Omega}$, and the following estimates which result from the "shallowness" hypothesis,

$$
\left|b_{\alpha \beta}\right|+\left|b_{\alpha \beta, \lambda}\right| \leq \delta
$$

where $\delta$ is a sufficiently small constant,

$$
\left|\epsilon_{\alpha \beta}(\vec{u})\right|_{0, \Omega} \geq C_{1}|\vec{u}|_{1, \Omega}, \quad \vec{u} \in H_{0}^{1}(\Omega)
$$


referred to as Korn's inequality for shells, see p. 39 of [1], and

$$
\left(u_{3 \mid \alpha \beta}, u_{3 \mid \alpha \beta}\right) \geq c\left|u_{3}\right|_{2, \Omega}^{2}, u_{3} \in H_{0}^{2}(\Omega)
$$

In order to derive our stability estimates, we introduce the discrete energy function, $E_{\gamma}^{h}(t)$ which replaces $E_{\gamma}(t)$ in Section 1.1.

$$
E_{\gamma}^{h}(t)=E_{p}^{h}(t)+E_{k}^{h, \gamma}(t)
$$

where

$$
\begin{aligned}
E_{k}^{h, \gamma}(t) & \equiv \frac{1}{2}\left[\left(\rho e a^{\alpha \beta} \dot{u}_{\alpha}^{h}, \dot{u}_{\beta}^{h}\right)+\left(\rho e \dot{u}_{3}^{h}, \dot{u}_{3}^{h}\right)+\gamma\left(\rho e \dot{u}_{3, \alpha}^{h}, \dot{u}_{3, \alpha}^{h}\right)\right] \\
E_{p}^{h}(t) & \equiv \frac{1}{2}\left[\left(e E^{\alpha \beta \lambda \mu} \gamma_{\alpha \beta}\left(\mathbf{u}^{h}\right), \gamma_{\lambda \mu}\left(\mathbf{u}^{h}\right)\right)+\left(\frac{e^{3}}{12} E^{\alpha \beta \lambda \mu} u_{3 \mid \alpha \beta}^{h}, u_{3 \mid \lambda \mu}^{h}\right)\right] .
\end{aligned}
$$

As mentioned earlier, the existence and uniqueness of solutions to the semidiscrete problem (1.5, 1.6) follows from a standard argument. Indeed, local (in time) existence and uniqueness follows from a fixed point argument (note that in the semidiscrete case the nonlinear terms are locally Lipschitz). The global (in time) existence follows from the a priori bound obtained by replacing $\mathbf{v}^{h}(t) \equiv \dot{\mathbf{u}}^{h}(t)$ in $(1.5,1.6)$ and integrating by parts in time to obtain the following stability estimate.

Lemma 2.1. Let $u^{h}(t)$ be a local solution to $(1.5,1.6)$. Then for $0 \leq t \leq T$

$$
E_{\gamma}^{h}(t) \leq C\left[E_{\gamma}^{h}(0)+|(\vec{p}, q)|^{2}\right] \leq C\left(E_{\gamma}(0),|(\vec{p}, q)|\right)
$$

This lemma will be used extensively in the development of the error estimate and leads naturally to the $a$ priori bound stated in the following lemma.

Lemma 2.2. Let $\mathbf{u}^{h}$ be a solution to $(1.5,1.6)$ with $\gamma \geq 0$. Assume that $\mathbf{u}_{0}^{h}$ is an approximation of the initial data $\mathbf{u}_{0}$ complying with the approximation properties in (1.4) and $\vec{p}, q \in L_{2}\left((0, T) ;\left[L_{2}(\Omega)\right]^{2} \times H^{-1}(\Omega)\right)$. Then the following bound applies for $0 \leq t \leq T$.

$$
\begin{aligned}
\left|u_{\alpha}^{h}(t)\right|_{1, \Omega}+\left|u_{3}^{h}(t)\right|_{2, \Omega} & +\left|\dot{u}_{\alpha}^{h}(t)\right|_{0, \Omega}+\left|\dot{u}_{3}^{h}(t)\right|_{0, \Omega}+\gamma\left|\dot{u}_{3}^{h}(t)\right|_{1, \Omega} \\
& \leq C\left(\left|u_{0_{\alpha}}^{h}\right|_{1, \Omega},\left|u_{0_{3}}^{h}\right|_{2, \Omega},\left|\dot{u}_{0_{\alpha}}^{h}\right|_{0, \Omega},\left|\dot{u}_{0_{3}}^{h}\right|_{0, \Omega}, \gamma\left|\dot{u}_{0_{3}}^{h}\right|_{1, \Omega},|(\vec{p}, q)|\right), \\
& \leq C\left(\left|u_{0_{\alpha}}\right|_{1, \Omega},\left|u_{0_{3}}\right|_{2, \Omega},\left|\dot{u}_{0_{\alpha}}\right|_{0, \Omega},\left|\dot{u}_{0_{3}}\right|_{0, \Omega}, \gamma\left|\dot{u}_{0_{3}}\right|_{1, \Omega},|(\vec{p}, q)|\right) .
\end{aligned}
$$

Proof. The proof exploits the shallowness assumption and a Korn's type of inequality recalled above. Since

$$
\left|\gamma_{\alpha \beta}\left(\mathbf{u}^{h}(t)\right)\right|_{0, \Omega}^{2}+\left|u_{3}^{h}(t)\right|_{2, \Omega}^{2} \leq C E_{p}^{h}(t)
$$

the shallowness hypothesis and Korn's inequality in (2.4), can be used to obtain

$$
\begin{aligned}
\left|u_{\alpha}^{h}\right|_{1, \Omega}^{2} \leq C\left|\epsilon_{\alpha \beta}\left(\vec{u}^{h}\right)\right|_{0, \Omega} & \leq C\left|\epsilon_{\alpha \beta}\left(\mathbf{u}^{h}\right)-b_{\alpha \beta} u_{3}^{h}\right|_{0, \Omega}^{2}+C\left|u_{3}^{h}\right|_{0, \Omega}^{2} \\
& \leq C\left|\gamma_{\alpha \beta}\left(\mathbf{u}^{h}\right)\right|_{0, \Omega}^{2}+C\left|u_{3}^{h}\right|_{W^{1,4}(\Omega)}^{4} \\
& \leq C\left|\gamma_{\alpha \beta}\left(\mathbf{u}^{h}\right)\right|_{0, \Omega}^{2}+C\left|u_{3}^{h}\right|_{2, \Omega}^{4} .
\end{aligned}
$$


By combining (2.6) and (2.7) we obtain

$$
\left|u_{\alpha}^{h}(t)\right|_{1, \Omega}^{2} \leq C\left[E_{p}^{h}(t)+\left(E_{p}^{h}(t)\right)^{2}\right]
$$

The remainder of the proof is straightforward.

Our next step is to derive a convenient form of an error equation which will be suitable for further analysis. To this end, we will use the following notation

$$
\gamma_{\alpha \beta}^{h} \equiv \gamma_{\alpha \beta}\left(\mathbf{u}^{h}\right), \quad \gamma_{\alpha \beta} \equiv \gamma_{\alpha \beta}(\mathbf{u}) \text { and } \tilde{\gamma}_{\alpha \beta}^{h} \equiv \gamma_{\alpha \beta}\left(\tilde{\mathbf{u}}^{h}\right)
$$

where $\tilde{\mathbf{u}}^{h}$ is a suitable approximation of the solution $\mathbf{u}$ satisfying the requirements in (1.4). Therefore, the time regularity of $\tilde{\mathbf{u}}^{h}$ is the same as that of $\mathbf{u}$. In addition, we define the following "error" expressions

$$
\begin{aligned}
\mathbf{e}^{h} & =\left(e_{\alpha}^{h}, e_{3}^{h}\right) \equiv\left(u_{\alpha}^{h}-\tilde{u}_{\alpha}^{h}, u_{3}^{h}-\tilde{u}_{3}^{h}\right) . \\
\mathcal{E}(t) & \equiv\left(\rho e a^{\alpha \beta} \dot{e}_{\alpha}^{h}, \dot{e}_{\beta}^{h}\right)+\left(\rho e \dot{e}_{3}^{h}, \dot{e}_{3}^{h}\right)+\gamma\left(\rho e \dot{e}_{3, \alpha}^{h}, \dot{e}_{3, \alpha}^{h}\right)+\left(e E^{\alpha \beta \lambda \mu}\left(\gamma_{\alpha \beta}^{h}-\tilde{\gamma}_{\alpha \beta}^{h}\right), \gamma_{\lambda \mu}^{h}-\tilde{\gamma}_{\lambda \mu}^{h}\right) \\
& +\left(\frac{e^{3}}{12} E^{\alpha \beta \lambda \mu} e_{3 \mid \alpha \beta}^{h}, e_{3 \mid \lambda \mu}^{h}\right) .
\end{aligned}
$$

Now subtracting the variational forms of the equations for $\mathbf{u}$ and $\mathbf{u}^{h}$ and adding and subtracting appropriate terms leads to the expressions

$$
\begin{gathered}
\left(\rho e a^{\alpha \beta} \ddot{e}_{\beta}^{h}, v_{\alpha}^{h}\right)+\left(e E^{\alpha \beta \lambda \mu}\left(\gamma_{\alpha \beta}^{h}-\tilde{\gamma}_{\alpha \beta}^{h}\right), \epsilon_{\lambda \mu}\left(\overrightarrow{v^{h}}\right)\right)=L_{1}\left(\mathbf{u}, \tilde{\mathbf{u}}^{h}, \mathbf{v}^{h}\right) \\
\left(\rho e \ddot{e}_{3}^{h}, v_{3}^{h}\right)+\gamma\left(\rho e \ddot{e}_{3, \alpha}^{h}, v_{3, \alpha}^{h}\right)+\left(\frac{e^{3}}{12} E^{\alpha \beta \lambda \mu} e_{3 \mid \alpha \beta}^{h}, v_{3 \mid \lambda \mu}^{h}\right) \\
+\left(e E^{\alpha \beta \lambda \mu}\left(\gamma_{\alpha \beta}^{h}-\tilde{\gamma}_{\alpha \beta}^{h}\right), u_{3, \lambda}^{h} v_{3, \mu}^{h}-b_{\lambda \mu} v_{3}^{h}\right)+\left(e E^{\alpha \beta \lambda \mu} \tilde{\gamma}_{\alpha \beta}^{h} e_{3, \lambda}^{h}, v_{3, \mu}^{h}\right)=L_{2}\left(\mathbf{u}, \tilde{\mathbf{u}}^{h}, \mathbf{v}^{h}\right)
\end{gathered}
$$

where

$$
L_{1}\left(\mathbf{u}, \tilde{\mathbf{u}}^{h}, \mathbf{v}^{h}\right) \equiv\left(\rho e a^{\alpha \beta} \ddot{e}_{\alpha}^{h}, v_{\beta}^{h}\right)+\left(e E^{\alpha \beta \lambda \mu}\left(\gamma_{\alpha \beta}-\tilde{\gamma}_{\alpha \beta}^{h}\right), \epsilon_{\lambda \mu}\left(\overrightarrow{v^{h}}\right)\right)
$$

and

$$
\begin{aligned}
L_{2}\left(\mathbf{u}, \tilde{\mathbf{u}}^{h}, \mathbf{v}^{h}\right) \equiv & -\left(e E^{\alpha \beta \lambda \mu}\left(\tilde{\gamma}_{\alpha \beta}^{h} \tilde{u}_{3, \lambda}^{h}-\gamma_{\alpha \beta} u_{3, \lambda}\right), v_{3, \mu}^{h}\right)+\left(\rho e \ddot{e}_{3}^{h}, v_{3}^{h}\right)+\gamma\left(\rho e \ddot{e}_{3, \alpha}^{h}, v_{3, \alpha}^{h}\right)+\left(\frac{e^{3}}{12} E^{\alpha \beta \lambda \mu} e_{3 \mid \alpha \beta}^{h}, v_{3 \mid \lambda \mu}^{h}\right) \\
& -\left(e E^{\alpha \beta \lambda \mu}\left(\gamma_{\alpha \beta}-\tilde{\gamma}_{\alpha \beta}^{h}\right), b_{\lambda \mu} v_{3}^{h}\right) .
\end{aligned}
$$

Note that both terms, $L_{1}$ and $L_{2}$ depend linearly on the test function $\mathbf{v}^{h}$. Taking advantage of the symmetric properties of $E^{\alpha \beta \lambda \mu}$ we obtain the relation

$$
\begin{aligned}
\frac{1}{2} \frac{\mathrm{d}}{\mathrm{d} t}\left(e E^{\alpha \beta \lambda \mu}\left(\gamma_{\alpha \beta}^{h}-\tilde{\gamma}_{\alpha \beta}^{h}\right), \gamma_{\lambda \mu}^{h}-\tilde{\gamma}_{\lambda \mu}^{h}\right)= & \left(e E^{\alpha \beta \lambda \mu}\left(\gamma_{\alpha \beta}^{h}-\tilde{\gamma}_{\alpha \beta}^{h}\right), \epsilon_{\lambda \mu}\left(\overrightarrow{\dot{u}}^{h}\right)-\epsilon_{\lambda \mu}\left(\overrightarrow{\tilde{u}}^{h}\right)-b_{\lambda \mu} \dot{e}_{3}^{h}\right) \\
& +\left(e E^{\alpha \beta \lambda \mu}\left(\gamma_{\alpha \beta}^{h}-\tilde{\gamma}_{\alpha \beta}^{h}\right), e_{3, \lambda}^{h} \dot{\tilde{u}}_{3, \mu}^{h}+u_{3, \lambda}^{h} \dot{e}_{3, \mu}^{h}\right) .
\end{aligned}
$$


In order to take advantage of this result, we add the expression $\left(e E^{\alpha \beta \lambda \mu}\left(\gamma_{\alpha \beta}^{h}-\tilde{\gamma}_{\alpha \beta}^{h}\right), e_{3, \lambda}^{h} \dot{\tilde{u}}_{3, \mu}^{h}\right)$ to both sides of (2.10) to obtain

$$
\begin{array}{r}
\left(\rho e \ddot{e}_{3}^{h}, v_{3}^{h}\right)+\gamma\left(\rho e \ddot{e}_{3, \alpha}^{h}, v_{3, \alpha}^{h}\right)+\left(\frac{e^{3}}{12} E^{\alpha \beta \lambda \mu} e_{3 \mid \alpha \beta}^{h}, v_{3 \mid \lambda \mu}^{h}\right)+\left(e E^{\alpha \beta \lambda \mu}\left(\gamma_{\alpha \beta}^{h}-\tilde{\gamma}_{\alpha \beta}^{h}\right), u_{3, \lambda}^{h} v_{3, \mu}^{h}-b_{\lambda \mu} v_{3}^{h}+e_{3, \lambda}^{h} \dot{\tilde{u}}_{3, \mu}^{h}\right) \\
=X+L_{2}+\left(e E^{\alpha \beta \lambda \mu}\left(\gamma_{\alpha \beta}^{h}-\tilde{\gamma}_{\alpha \beta}^{h}\right), e_{3, \lambda}^{h} \dot{\tilde{u}}_{3, \mu}^{h}\right)
\end{array}
$$

where

$$
-X\left(\mathbf{v}^{h}\right) \equiv\left(e E^{\alpha \beta \lambda \mu} \tilde{\gamma}_{\alpha \beta}^{h} e_{3, \lambda}^{h}, v_{3, \mu}^{h}\right)
$$

Now we set $\mathbf{v}^{h}=\dot{\mathbf{u}}^{h}-\dot{\tilde{\mathbf{u}}}^{h}=\mathbf{e}^{h}$ in (2.9) and (2.12) and add the two equations. After accounting for (2.11) and performing several calculations, we obtain

$$
\frac{1}{2} \frac{\mathrm{d}}{\mathrm{d} t} \mathcal{E}(t)=L_{1}\left(\mathbf{u}, \tilde{\mathbf{u}}^{h}, \dot{\mathbf{e}}^{h}\right)+X\left(\dot{\mathbf{e}}^{h}\right)+L_{2}\left(\mathbf{u}, \tilde{\mathbf{u}}^{h}, \dot{\mathbf{e}}^{h}\right)+\left(e E^{\alpha \beta \lambda \mu}\left(\gamma_{\alpha \beta}^{h}-\tilde{\gamma}_{\alpha \beta}^{h}\right), e_{3, \lambda}^{h} \dot{\tilde{u}}_{3, \mu}^{h}\right)
$$

Integrating (2.13) from 0 to $t$ gives the following error equation:

$$
\mathcal{E}(t)=\mathcal{E}(0)+2 \int_{0}^{t}\left(L_{1}\left(\mathbf{u}, \tilde{\mathbf{u}}^{h}, \dot{\mathbf{e}}^{h}\right)+L_{2}\left(\mathbf{u}, \tilde{\mathbf{u}}^{h}, \dot{\mathbf{e}}^{h}\right) \mathrm{d} \tau+2 \int_{0}^{t} X\left(\dot{\mathbf{e}}^{h}\right) \mathrm{d} \tau+2 \int_{0}^{t}\left(e E^{\alpha \beta \lambda \mu}\left(\gamma_{\alpha \beta}^{h}-\tilde{\gamma}_{\alpha \beta}^{h}\right), e_{3, \lambda}^{h} \dot{\tilde{u}}_{3, \mu}^{h}\right) \mathrm{d} t\right.
$$

We will proceed by developing estimates for the terms on the right-hand side of (2.14). As suggested by the notation, the contribution of $L_{1}$ and $L_{2}$ will be expressed in terms of the error in the approximation. The contribution of the last term in (2.14) will be controlled by exploiting the coercivity properties of the error function $\mathcal{E}(t)$. The most critical part is the analysis of $X$, which involves the higher order nonlinear terms combined with time derivatives $\dot{\mathbf{e}}^{h}$ of the error. While it is reasonably straightforward to derive the error estimates for the term, $X$ which blow up with $\gamma \rightarrow 0$, the main task of this paper is to obtain these estimates independent on the parameter $\gamma$, including the critical case $\gamma=0$. This forces upon additional technical difficulties which will be dealt with next.

\section{The estimates}

Our main effort is directed toward error estimates obtained for each integral term in equation (2.14). The proof is divided into several Lemmas and Propositions, which provide partial results. The most important is Lemma 3.2 which gives the error estimates for the solutions in terms of the error estimates for the interpolants of the original continuous solution. The estimates for the interpolants are provided in Lemma 3.3. Combining the results of Lemma 3.2 with Lemma 3.3 gives the final result stated in the main Theorem 1.2.

We begin by stating the following lemma which will be used later in this exposition.

Lemma 3.1. Let $f$ be a given function with the regularity $f \in C\left([0, T] ; H^{2}(\Omega)\right) \cap H^{1}\left((0, T) ; L_{2}(\Omega)\right)$. For all $\epsilon>0$ and $T>0$, there exists a constant $C_{T, \epsilon}$ such that for all $t \leq T$

$$
|f(t)|_{W^{1,4}(\Omega)}^{2} \leq \epsilon|f(t)|_{2, \Omega}^{2}+C_{T, \epsilon} \int_{0}^{t}\left|\frac{\mathrm{d}}{\mathrm{d} \tau} f(\tau)\right|_{0, \Omega}^{2} \mathrm{~d} \tau+C_{\epsilon}|f(0)|_{0, \Omega}^{2}
$$


Proof of Lemma 3.1. By Sobolev embedding, $H^{\frac{1}{2}}(\Omega) \subset L_{4}(\Omega)$ which implies that

$$
|f(t)|_{W^{1,4}(\Omega)} \leq C|f(t)|_{\frac{3}{2}, \Omega} .
$$

Using the interpolation inequality, e.g. Theorem 9.6 of [18]

$$
|f|_{s, \Omega} \leq|f|_{s_{1}, \Omega}^{1-\theta}|f|_{s_{2}, \Omega}^{\theta} \text { where } s=(1-\theta) s_{1}+\theta s_{2} ; 0 \leq \theta \leq 1,
$$

with $\theta=1 / 4, s_{1}=2$, and $s_{2}=0$, then

$$
|f(t)|_{\frac{3}{2}, \Omega} \leq C|f(t)|_{0, \Omega}^{\frac{1}{4}}|f(t)|_{2, \Omega}^{\frac{3}{4}} .
$$

Now apply Young's inequality given in [23] with $p=4$ and $q=4 / 3$ to obtain

$$
|f(t)|_{\frac{3}{2}, \Omega} \leq C_{\epsilon}|f(t)|_{0, \Omega}+\epsilon|f(t)|_{2, \Omega}
$$

where $\epsilon>0$ is arbitrary and $C_{\epsilon}=\mathcal{O}\left(\frac{1}{\epsilon}\right)$. Coupling this result with (3.1) shows that

$$
|f(t)|_{W^{1,4}(\Omega)} \leq C_{\epsilon}|f(t)|_{0, \Omega}+\epsilon|f(t)|_{2, \Omega} .
$$

We square both sides of this inequality and rescale epsilon to obtain (see also [22])

$$
|f(t)|_{W^{1,4}(\Omega)}^{2} \leq C_{\epsilon}|f(t)|_{0, \Omega}^{2}+\epsilon|f(t)|_{2, \Omega}^{2} .
$$

By Sobolev embedding $H^{1}(0, T ; \mathcal{X}) \subset C([0, T] ; \mathcal{X}) \forall T>0$ and the Fundamental Theorem of Calculus

$$
|f(t)|_{0, \Omega}^{2}=\left|\int_{0}^{t} \frac{\mathrm{d}}{\mathrm{d} \tau} f(\tau) \mathrm{d} \tau+f(0)\right|_{0, \Omega}^{2} \leq C_{T} \int_{0}^{t}\left|\frac{\mathrm{d}}{\mathrm{d} \tau} f(\tau)\right|_{0, \Omega}^{2} \mathrm{~d} \tau+|f(0)|_{0, \Omega}^{2}
$$

Combining $(3.4,3.3)$ gives the result of the lemma.

\subsection{First estimate}

The main goal of this subsection is to prove the following error estimate

Lemma 3.2. For all $\epsilon_{0}$ and $T>0$, there exists $C_{T, \epsilon_{0}}>0$ such that the following estimate is valid

$$
\left|\dot{e}_{\alpha}^{h}(t)\right|_{0, \Omega}^{2}+\left|\dot{e}_{3}^{h}(t)\right|_{0, \Omega}^{2}+\gamma\left|\dot{e}_{3}^{h}(t)\right|_{1, \Omega}^{2}+\left|e_{3}^{h}(t)\right|_{2, \Omega}^{2}+\left|e_{\alpha}^{h}(t)\right|_{1, \Omega}^{2} \leq C_{T, \epsilon_{0}}\left(E_{\gamma}(0),|(\vec{p}, q)|,\left|\dot{u}_{3}\right|_{1+\epsilon_{0}, \infty}\right) Y(t)
$$

where we have made the following definitions

$$
Y(t) \equiv Y_{u}(t)+Y_{0}
$$

with

$$
\begin{aligned}
Y_{u}(t) \equiv & \left(\int_{0}^{t}\left|\dot{\gamma}_{\alpha \beta}-\dot{\tilde{\gamma}}_{\alpha \beta}^{h}\right|_{0, \Omega} \mathrm{d} \tau\right)^{2}+\int_{0}^{t}\left[\left|\ddot{u}_{\alpha}-\ddot{\tilde{u}}_{\alpha}^{h}\right|_{0, \Omega}^{2}+\left|\ddot{u}_{3}-\ddot{\tilde{u}}_{3}^{h}\right|_{0, \Omega}^{2}+\gamma\left|\ddot{u}_{3}-\ddot{\tilde{u}}_{3}^{h}\right|_{1, \Omega}^{2}+\left|\gamma_{\alpha \beta}-\tilde{\gamma}_{\alpha \beta}^{h}\right|_{0, \Omega}^{2}\right] \mathrm{d} \tau \\
& +\left(\int_{0}^{t}\left|\dot{u}_{3}-\dot{\tilde{u}}_{3}^{h}\right|_{2, \Omega} \mathrm{d} \tau\right)^{2}+\left(\int_{0}^{t}\left|\frac{\mathrm{d}}{\mathrm{d} t}\left(\tilde{\gamma}_{\alpha \beta}^{h} \tilde{u}_{3, \lambda}^{h}-\gamma_{\alpha \beta} u_{3, \lambda}\right)\right|_{-1, \Omega} \mathrm{d} \tau\right)^{2}
\end{aligned}
$$


and

$$
\begin{aligned}
Y_{0} \equiv & \sup _{0 \leq \tau \leq t}\left|\tilde{\gamma}_{\alpha \beta}^{h}(\tau)\right|_{0, \Omega}^{2}\left|e_{3}^{h}(0)\right|_{2, \Omega}^{2}+\mathcal{E}(0)+\left|\tilde{\gamma}_{\alpha \beta}^{h} \tilde{u}_{3, \lambda}^{h}(0)-\gamma_{\alpha \beta} u_{3, \lambda}(0)\right|_{-1, \Omega}^{2}+\left|e_{\alpha}^{h}(0)\right|_{1, \Omega}\left|\gamma_{\alpha \beta}(0)-\tilde{\gamma}_{\alpha \beta}^{h}(0)\right|_{0, \Omega} \\
& +\left|e_{3}^{h}(0)\right|_{2, \Omega}\left|u_{3}(0)-\tilde{u}_{3}^{h}(0)\right|_{2, \Omega} .
\end{aligned}
$$

Proof of Lemma 3.2. The proof proceeds through several steps. The basic idea is to obtain the estimates for each term in the error equation (2.14). We begin with a straightforward estimate involving the fourth term on the right-hand side in (2.14).

Proposition 3.1. For any $\epsilon>0$,

$$
\int_{0}^{t}\left(e E^{\alpha \beta \lambda \mu}\left(\gamma_{\alpha \beta}^{h}-\tilde{\gamma}_{\alpha \beta}^{h}\right), e_{3, \lambda}^{h} \dot{\tilde{u}}_{3, \mu}^{h}\right) \mathrm{d} \tau \leq C_{\epsilon} \sup _{0 \leq \tau \leq t}\left|\dot{\tilde{u}}_{3}^{h}(t)\right|_{W^{1,2+\epsilon}(\Omega)}\left[\int_{0}^{t}\left[\left|\gamma_{\alpha \beta}^{h}-\tilde{\gamma}_{\alpha \beta}^{h}\right|_{0, \Omega}^{2}+\left|e_{3}^{h}\right|_{2, \Omega}^{2}\right] \mathrm{d} \tau\right] .
$$

Proof. The Cauchy-Schwarz and Holder's inequalities guarantee that

$$
\int_{0}^{t}\left(e E^{\alpha \beta \lambda \mu}\left(\gamma_{\alpha \beta}^{h}-\tilde{\gamma}_{\alpha \beta}^{h}\right), e_{3, \lambda}^{h} \dot{\tilde{u}}_{3, \mu}^{h}\right) \mathrm{d} \tau \leq C \int_{0}^{t}\left|\gamma_{\alpha \beta}^{h}-\tilde{\gamma}_{\alpha \beta}^{h}\right|_{0, \Omega}\left|e_{3}^{h}\right|_{W^{1,2 p}(\Omega)}\left|\dot{\tilde{u}}_{3}^{h}\right|_{W^{1,2 \bar{p}}(\Omega)} \mathrm{d} \tau
$$

where $1 / p+1 / \bar{p}=1$. Applying the Sobolev embedding $H^{2}(\Omega) \subset W^{1,2 p}(\Omega)$ for $p>1 / 2$ and the arithmeticgeometric mean inequality to this result provides the inequality

$$
\int_{0}^{t}\left(e E^{\alpha \beta \lambda \mu}\left(\gamma_{\alpha \beta}^{h}-\tilde{\gamma}_{\alpha \beta}^{h}\right), e_{3, \lambda}^{h} \dot{\tilde{u}}_{3, \mu}^{h}\right) \mathrm{d} \tau \leq C_{\epsilon} \int_{0}^{t}\left[\left|\gamma_{\alpha \beta}^{h}-\tilde{\gamma}_{\alpha \beta}^{h}\right|_{0, \Omega}^{2}+\left|e_{3}^{h}\right|_{2, \Omega}^{2}\right]\left|\dot{\tilde{u}}_{3}^{h}\right|_{W^{1,2+\epsilon}(\Omega)} \mathrm{d} \tau
$$

which implies the conclusion in Proposition 3.1.

\subsubsection{Estimate for $X$}

Now we are prepared to derive an estimate for $\left.X\left(\dot{\mathbf{e}}^{h}\right)\right)$ as stated in the following proposition.

Proposition 3.2. Let $\epsilon>0$ and $\epsilon_{0}>0$ be arbitrary (small) and let $0 \leq t \leq T$. Then the following estimate holds:

$$
\begin{aligned}
\int_{0}^{t}\left|X\left(\dot{\mathbf{e}}^{h}\right)\right| \mathrm{d} \tau \leq & \epsilon\left|e_{3}^{h}(t)\right|_{2, \Omega}^{2}+C_{T, \epsilon} \sup _{0 \leq t \leq T}\left|\tilde{\gamma}_{\alpha \beta}^{h}(t)\right|_{0, \Omega}\left[\left|u_{3}^{h}(0)-\tilde{u}_{3}^{h}(0)\right|_{2, \Omega}^{2}+\int_{0}^{t}\left|\dot{e}_{3}^{h}(\tau)\right|_{0, \Omega}^{2} \mathrm{~d} \tau\right] \\
& +C_{\epsilon_{0}} \sup _{0 \leq t \leq T}\left|\dot{\tilde{\gamma}}_{\alpha \beta}^{h}(t)\right|_{L_{1+\epsilon_{0}}(\Omega)} \int_{0}^{t}\left|e_{3}^{h}(\tau)\right|_{2, \Omega}^{2} \mathrm{~d} \tau .
\end{aligned}
$$

Proof. Exploiting the symmetry of $E^{\alpha \beta \lambda \mu}$ we obtain

$$
\int_{0}^{t}\left(e E^{\alpha \beta \lambda \mu} \tilde{\gamma}_{\alpha \beta}^{h} e_{3, \lambda}^{h}, \dot{e}_{3, \mu}^{h}\right) \mathrm{d} \tau=\int_{0}^{t}\left(e E^{\alpha \beta \lambda \mu} \tilde{\gamma}_{\alpha \beta}^{h}, \frac{\mathrm{d}}{\mathrm{d} t}\left[e_{3, \lambda}^{h} e_{3, \mu}^{h}\right]\right) \mathrm{d} \tau .
$$


Thus, by integrating by parts we have

$$
\begin{aligned}
\int_{0}^{t}\left(e E^{\alpha \beta \lambda \mu} \tilde{\gamma}_{\alpha \beta}^{h} e_{3, \lambda}^{h}, \dot{e}_{3, \mu}^{h}\right) \mathrm{d} \tau= & \frac{1}{2}\left(e E^{\alpha \beta \lambda \mu} \tilde{\gamma}_{\alpha \beta}^{h}(t), e_{3, \lambda}^{h}(t) e_{3, \mu}^{h}(t)\right) \\
& -\frac{1}{2}\left(e E^{\alpha \beta \lambda \mu} \tilde{\gamma}_{\alpha \beta}^{h}(0), e_{3, \lambda}^{h}(0) e_{3, \mu}^{h}(0)\right) \\
& -\frac{1}{2} \int_{0}^{t}\left(e E^{\alpha \beta \lambda \mu} \dot{\tilde{\gamma}}_{\alpha \beta}^{h}(\tau), e_{3, \lambda}^{h}(\tau) e_{3, \mu}^{h}(\tau)\right) \mathrm{d} \tau .
\end{aligned}
$$

Since $E^{\alpha \beta \lambda \mu}$ is bounded pointwise, (3.7), Holder's inequality and Sobolev embedding justify the estimate

$$
\begin{aligned}
\int_{0}^{t}\left|X\left(\dot{\mathbf{e}}^{h}\right)\right| \mathrm{d} \tau \leq & C\left[\left|\tilde{\gamma}_{\alpha \beta}^{h}(0)\right|_{0, \Omega}\left|u_{3}^{h}(0)-\tilde{u}_{3}^{h}(0)\right|_{W^{1,4}(\Omega)}^{2}+\left|\tilde{\gamma}_{\alpha \beta}^{h}(t)\right|_{0, \Omega}\left|e_{3}^{h}(t)\right|_{W^{1,4}(\Omega)}^{2}\right] \\
& +C \int_{0}^{t}\left|\dot{\tilde{\gamma}}_{\alpha \beta}^{h}(\tau)\right|_{L_{\bar{p}}(\Omega)}\left|e_{3}^{h}(\tau)\right|_{W^{1, p}(\Omega)}^{2} \mathrm{~d} \tau
\end{aligned}
$$

where $p$ can be taken to be arbitrarily large with $1 / \bar{p}+1 / p=1$.

We now apply Lemma 3.1 with $f$ replaced by the error function $e_{3}^{h}(t)$. This yields: For all $\epsilon>0$ and $T>0$, there exists a constant $C_{T, \epsilon}$ such that for all $t \leq T$

$$
\left|e_{3}^{h}\right|_{W^{1,4}(\Omega)}^{2} \leq \epsilon\left|e_{3}^{h}(t)\right|_{2, \Omega}^{2}+C_{T, \epsilon} \int_{0}^{t}\left|\dot{e}_{3}^{h}(\tau)\right|_{0, \Omega}^{2} d \tau+C_{\epsilon}\left|e_{3}^{h}(0)\right|_{0, \Omega}^{2}
$$

Applying the result of inequality (3.9) to the second term on the right-hand side of (3.8) and recalling Sobolev's embedding, $W^{1, p}(\Omega) \supset H^{2}(\Omega)$ for any $p>1$ and taking $p=1+\epsilon_{0}$ with $\epsilon_{0}>0$, we can conclude from (3.8) that

$$
\begin{aligned}
\int_{0}^{t}\left|X\left(\dot{\mathbf{e}}^{h}\right)\right| \mathrm{d} \tau \leq & C \sup _{0 \leq t \leq T}\left|\tilde{\gamma}_{\alpha \beta}^{h}(t)\right|_{0, \Omega}\left[\epsilon\left|e_{3}^{h}(t)\right|_{2, \Omega}^{2}+C_{T, \epsilon} \int_{0}^{t}\left|\dot{e}_{3}^{h}(\tau)\right|_{0, \Omega}^{2} \mathrm{~d} \tau+C_{\epsilon}\left|e_{3}^{h}(0)\right|_{2, \Omega}^{2}\right] \\
& +C_{\epsilon_{0}} \sup _{0 \leq t \leq T}\left|\dot{\tilde{\gamma}}_{\alpha, \beta}^{h}(t)\right|_{L_{1+\epsilon_{0}}(\Omega)} \int_{0}^{t}\left|e_{3}^{h}(\tau)\right|_{2, \Omega}^{2} \mathrm{~d} \tau
\end{aligned}
$$

where $\epsilon_{0}, \epsilon$ are arbitrary. Finally, rescaling $\epsilon \sim \epsilon C \sup _{0 \leq t \leq T}\left|\tilde{\gamma}_{\alpha \beta}^{h}(t)\right|_{0, \Omega}$ leads to the desired estimate in Proposition 3.2.

3.1.2. Estimates for $L_{1}$ and $L_{2}$

Our next step is to estimate terms described by $L_{1}$ and $L_{2}$. This is done in the following two propositions.

Proposition 3.3. For any $\epsilon>0$ there exist a constant $C_{\epsilon}$ such that for all $t \leq T$ :

$$
\begin{aligned}
\int_{0}^{t} L_{1}\left(\mathbf{u}, \tilde{\mathbf{u}}^{h}, \dot{\mathbf{e}}^{h} \mathrm{~d} \tau \leq\right. & \left.\epsilon \sup _{0 \leq \tau \leq t}\left|e_{\alpha}^{h}(\tau)\right|_{1, \Omega}^{2}+C_{\epsilon}\left(\int_{0}^{t}\left|\dot{\gamma}_{\alpha \beta}-\dot{\tilde{\gamma}}_{\alpha \beta}^{h}\right|_{0, \Omega} \mathrm{d} \tau\right)^{2}+\left.C \int_{0}^{t}\left[\left|\dot{e}_{\alpha}^{h}\right|_{0, \Omega}^{2}+\mid \ddot{u}_{\alpha}-\ddot{\tilde{u}}_{\alpha}^{h}\right)\right|_{0, \Omega} ^{2}\right] \mathrm{d} \tau \\
& +C\left|u_{\alpha}^{h}(0)-\tilde{u}_{\alpha}^{h}(0)\right|_{1, \Omega}\left|\gamma_{\alpha \beta}(0)-\tilde{\gamma}_{\alpha \beta}^{h}(0)\right|_{0, \Omega} .
\end{aligned}
$$

Proof. Recall that

$$
L_{1}\left(\mathbf{u}, \tilde{\mathbf{u}}^{h}, \dot{\mathbf{e}}^{h}\right) \equiv\left(\rho e a^{\alpha \beta}\left(\ddot{u}_{\alpha}-\ddot{\tilde{u}}_{\alpha}^{h}\right), \dot{e}_{\beta}^{h}\right)+\left(e E^{\alpha \beta \lambda \mu}\left(\gamma_{\alpha \beta}-\tilde{\gamma}_{\alpha \beta}^{h}\right), \epsilon_{\lambda \mu}\left(\dot{\vec{u}}^{h}-\dot{\vec{u}}^{h}\right)\right.
$$


The estimate for the first term in $L_{1}$ is straightforward. Indeed,

$$
\int_{0}^{t}\left(\rho e a^{\alpha \beta}\left(\ddot{u}_{\beta}-\ddot{\tilde{u}}_{\beta}^{h}\right), \dot{e}_{\alpha}^{h}\right) \mathrm{d} \tau \leq C \int_{0}^{t}\left|\ddot{u}_{\beta}-\ddot{\tilde{u}}_{\beta}^{h}\right|_{0, \Omega}^{2} \mathrm{~d} \tau+C \int_{0}^{t}\left|\dot{e}_{\alpha}^{h}\right|_{0, \Omega}^{2} \mathrm{~d} \tau .
$$

In order to estimate the second term, we integrate by parts to obtain

$$
\begin{gathered}
\int_{0}^{t}\left(e E^{\alpha \beta \lambda \mu}\left(\gamma_{\alpha \beta}-\tilde{\gamma}_{\alpha \beta}^{h}\right), \epsilon_{\lambda \mu}\left(\dot{\vec{u}}^{h}-\dot{\vec{u}}^{h}\right)\right) \mathrm{d} \tau=\left(e E^{\alpha \beta \lambda \mu}\left(\gamma_{\alpha \beta}(t)-\tilde{\gamma}_{\alpha \beta}^{h}(t)\right), \epsilon_{\lambda \mu}\left(\vec{u}^{h}(t)-\overrightarrow{\vec{u}}^{h}(t)\right)\right) \\
-\left(e E^{\alpha \beta \lambda \mu}\left(\gamma_{\alpha \beta}(0)-\tilde{\gamma}_{\alpha \beta}^{h}(0)\right), \epsilon_{\lambda \mu}\left(\vec{u}^{h}(0)-\overrightarrow{\tilde{u}}^{h}(0)\right)\right)-\int_{0}^{t}\left(e E^{\alpha \beta \lambda \mu}\left(\dot{\gamma}_{\alpha \beta}-\dot{\tilde{\gamma}}_{\alpha \beta}^{h}\right), \epsilon_{\lambda \mu}\left(\vec{u}^{h}-\overrightarrow{\tilde{u}}^{h}\right)\right) \mathrm{d} \tau \\
\left.\leq \epsilon \sup _{0 \leq \tau \leq t}\left|u_{\alpha}^{h}(\tau)-\tilde{u}_{\alpha}^{h}(\tau)\right|_{1, \Omega}^{2}+\frac{C}{\epsilon}\left|\gamma_{\alpha \beta}(t)-\tilde{\gamma}_{\alpha \beta}^{h}(t)\right|_{0, \Omega}^{2}+\frac{C}{\epsilon}\left(\int_{0}^{t}\left|\dot{\gamma}_{\alpha \beta}-\dot{\tilde{\gamma}}_{\alpha \beta}^{h}\right|_{0, \Omega} \mathrm{d} \tau\right)\right)^{2} \\
+C\left|u_{\alpha}^{h}(0)-\tilde{u}_{\alpha}^{h}(0)\right|_{1, \Omega}\left|\gamma_{\alpha \beta}(0)-\tilde{\gamma}_{\alpha \beta}^{h}(0)\right|_{0, \Omega} \\
\leq \epsilon \sup _{0 \leq \tau \leq t}\left|u_{\alpha}^{h}(\tau)-\tilde{u}_{\alpha}^{h}(\tau)\right|_{1, \Omega}^{2}+\frac{C}{\epsilon}\left(\int_{0}^{t}\left|\dot{\gamma}_{\alpha \beta}-\dot{\tilde{\gamma}}_{\alpha \beta}^{h}\right|_{0, \Omega} \mathrm{d} \tau\right)^{2}+C\left|u_{\alpha}^{h}(0)-\tilde{u}_{\alpha}^{h}(0)\right|_{1, \Omega}\left|\gamma_{\alpha \beta}(0)-\tilde{\gamma}_{\alpha \beta}^{h}(0)\right|_{0, \Omega} . \quad(3.1
\end{gathered}
$$

The inequality in (3.12) follows from the embedding $W^{1,1}(0, T) \subset C(0, T)$. Combining $(3.11,3.12)$ provides the result stated in the Proposition.

For the term $L_{2}$ we have the following Proposition.

Proposition 3.4. For any $\epsilon>0$ and $t \geq 0$,

$$
\begin{aligned}
\int_{0}^{t} L_{2}\left(\mathbf{u}, \tilde{\mathbf{u}}^{h}, \dot{\mathbf{e}}^{h}\right) \mathrm{d} \tau \leq & \epsilon \sup _{0 \leq \tau \leq t}\left|e_{3}^{h}(\tau)\right|_{2, \Omega}^{2}+C_{\epsilon}\left(\int_{0}^{t}\left|\dot{u}_{3}-\dot{\tilde{u}}_{3}^{h}\right|_{2, \Omega} \mathrm{d} \tau\right)^{2}+C \int_{0}^{t}\left[\left|\dot{e}_{3}^{h}\right|_{0, \Omega}^{2}+\gamma\left|\dot{e}_{3}^{h}\right|_{1, \Omega}^{2}\right] \mathrm{d} \tau \\
& +C_{\epsilon}\left(\int_{0}^{t}\left|\frac{\mathrm{d}}{\mathrm{d} t}\left(\tilde{\gamma}_{\alpha \beta}^{h} \tilde{u}_{3, \lambda}^{h}-\gamma_{\alpha \beta} u_{3, \lambda}\right)\right|_{-1, \Omega} \mathrm{d} \tau\right)^{2} \\
& +C \int_{0}^{t}\left[\left|\ddot{u}_{3}-\ddot{\tilde{u}}_{3}^{h}\right|_{0, \Omega}^{2}+\gamma\left|\ddot{u}_{3}-\ddot{\tilde{u}}_{3}^{h}\right|_{1, \Omega}^{2}+\left|\gamma_{\alpha \beta}-\tilde{\gamma}_{\alpha \beta}^{h}\right|_{0, \Omega}^{2}\right] \mathrm{d} \tau \\
& +C\left|\left(u_{3}^{h}-\tilde{u}_{3}^{h}\right)(0)\right|_{2, \Omega}^{2}+C_{\epsilon}\left|\tilde{\gamma}_{\alpha \beta}^{h} \tilde{u}_{3, \lambda}^{h}(0)-\gamma_{\alpha \beta} u_{3, \lambda}(0)\right|_{-1, \Omega}^{2} .
\end{aligned}
$$

Proof.

Step 1. Time derivatives:

Recall

$$
\begin{aligned}
L_{2}\left(\mathbf{u}, \tilde{\mathbf{u}}^{h}, \dot{\mathbf{e}}^{h}\right) \equiv & -\left(e E^{\alpha \beta \lambda \mu}\left(\tilde{\gamma}_{\alpha \beta}^{h} \tilde{u}_{3, \lambda}^{h}-\gamma_{\alpha \beta} u_{3, \lambda}\right), \dot{e}_{3, \mu}^{h}\right)+\left(\rho e\left(\ddot{u}_{3}-\ddot{\tilde{u}}_{3}^{h}\right), \dot{e}_{3}^{h}\right)+\gamma\left(\rho e\left(\ddot{u}_{3, \alpha}-\ddot{\tilde{u}}_{3, \alpha}^{h}\right), \dot{e}_{3, \alpha}^{h}\right) \\
& +\left(\frac{e^{3}}{12} E^{\alpha \beta \lambda \mu}\left(u_{3 \mid \alpha \beta}-\tilde{u}_{3 \mid \alpha \beta}^{h}\right), \dot{e}_{3 \mid \lambda \mu}^{h}\right)-\left(e E^{\alpha \beta \lambda \mu}\left(\gamma_{\alpha \beta}-\tilde{\gamma}_{\alpha \beta}^{h}\right), b_{\lambda \mu} \dot{e}_{3}^{h}\right) .
\end{aligned}
$$

By applying the Cauchy-Schwarz and arithmetic-geometric mean inequalities to the terms which are second order in time, the following estimate results.

$$
\int_{0}^{t}\left[\left(\ddot{u}_{3}-\ddot{\tilde{u}}_{3}^{h}, \dot{e}_{3}^{h}\right)+\gamma\left(\ddot{u}_{3, \alpha}-\ddot{\tilde{u}}_{3, \alpha}^{h}, \dot{e}_{3, \alpha}^{h}\right)\right] \mathrm{d} \tau \leq \int_{0}^{t}\left[\left|\dot{e}_{3}^{h}\right|_{0, \Omega}^{2}+\gamma\left|\dot{e}_{3}^{h}\right|_{1, \Omega}^{2}+\left|\ddot{u}_{3}-\ddot{\tilde{u}}_{3}^{h}\right|_{0, \Omega}^{2}+\gamma\left|\ddot{u}_{3}-\ddot{\tilde{u}}_{3}^{h}\right|_{1, \Omega}^{2}\right] \mathrm{d} \tau
$$


Step 2. Biharmonic terms:

Next, the biharmonic terms are integrated by parts to get

$$
\begin{aligned}
\int_{0}^{t}\left(\frac{e^{3}}{12} E^{\alpha \beta \lambda \mu}\left(u_{3 \mid \alpha \beta}-\tilde{u}_{3 \mid \alpha \beta}^{h}\right), \dot{e}_{3 \mid \lambda \mu}^{h}\right) \mathrm{d} \tau= & -\int_{0}^{t}\left(\frac{e^{3}}{12} E^{\alpha \beta \lambda \mu}\left(\dot{u}_{3 \mid \alpha \beta}-\dot{\tilde{u}}_{3 \mid \alpha \beta}^{h}\right), e_{3 \mid \lambda \mu}^{h}\right) \mathrm{d} \tau \\
& +\left.\left(\frac{e^{3}}{12} E^{\alpha \beta \lambda \mu}\left(u_{3 \mid \alpha \beta}-\tilde{u}_{3 \mid \alpha \beta}^{h}\right), e_{3 \mid \lambda \mu}^{h}\right)\right|_{0} ^{t} .
\end{aligned}
$$

Using this result and analogous arguments to those in the previous lemma including the embedding $W^{1,1}(0, T) \subset$ $C(0, T)$ applied to the last term, we have

$$
\begin{aligned}
\int_{0}^{t}\left(\frac{e^{3}}{12} E^{\alpha \beta \lambda \mu}\left(u_{3 \mid \alpha \beta}-\tilde{u}_{3 \mid \alpha \beta}^{h}\right), \dot{e}_{3 \mid \lambda \mu}^{h}\right) \mathrm{d} \tau \leq & \epsilon \sup _{0 \leq \tau \leq t}\left|e_{3}^{h}(\tau)\right|_{2, \Omega}^{2}+C_{\epsilon}\left(\int_{0}^{t}\left|\dot{u}_{3}-\dot{\tilde{u}}_{3}^{h}\right|_{2, \Omega} \mathrm{d} \tau\right)^{2} \\
& +C\left|\left(u_{3}-\tilde{u}_{3}^{h}\right)(0)\right|_{2, \Omega}\left|e_{3}^{h}(0)\right|_{2, \Omega} .
\end{aligned}
$$

\section{Step 3. Nonlinear terms:}

Integrating the nonlinear terms by parts, using duality pairings and the inclusions $H^{1}(\Omega) \subset L_{p}(\Omega) ; p \leq \infty$ and $W^{1,1}(0, T) \subset C(0, T)$ yields

$$
\begin{aligned}
\int_{0}^{t}\left(e E^{\alpha \beta \lambda \mu}\left(\tilde{\gamma}_{\alpha \beta}^{h} \tilde{u}_{3, \lambda}^{h}-\gamma_{\alpha \beta} u_{3, \lambda}\right), \dot{e}_{3, \mu}^{h}\right) \mathrm{d} \tau= & -\int_{0}^{t}\left(e E^{\alpha \beta \lambda \mu} \frac{\mathrm{d}}{\mathrm{d} t}\left(\tilde{\gamma}_{\alpha \beta}^{h} \tilde{u}_{3, \lambda}^{h}-\gamma_{\alpha \beta} u_{3, \lambda}\right), e_{3, \mu}^{h}\right) \\
& +\left.\left(e E^{\alpha \beta \lambda \mu}\left(\tilde{\gamma}_{\alpha \beta}^{h} \tilde{u}_{3, \lambda}^{h}-\gamma_{\alpha \beta} u_{3, \lambda}\right), e_{3, \mu}^{h}\right)\right|_{0} ^{t} \\
\leq & \epsilon \sup _{0 \leq \tau \leq T}\left|e_{3}^{h}\right|_{2, \Omega}^{2}+\frac{C}{\epsilon}\left(\int_{0}^{t}\left|\frac{\mathrm{d}}{\mathrm{d} t}\left(\tilde{\gamma}_{\alpha \beta}^{h} \tilde{u}_{3, \lambda}^{h}-\gamma_{\alpha \beta} u_{3, \lambda}\right)\right|_{-1, \Omega} \mathrm{d} \tau\right)^{2} \\
& +C_{\epsilon}\left|\tilde{\gamma}_{\alpha \beta}^{h} \tilde{u}_{3, \lambda}^{h}(0)-\gamma_{\alpha \beta} u_{3, \lambda}(0)\right|_{-1, \Omega}^{2} .
\end{aligned}
$$

Step 4. The last term in $L_{2}$ :

Through direct application of the Cauchy-Schwarz and arithmetic-geometric mean inequalities, the remaining term in $L_{2}$ is be estimated as follows.

$$
\int_{0}^{t}\left(e E^{\alpha \beta \lambda \mu}\left(\gamma_{\alpha \beta}-\tilde{\gamma}_{\alpha \beta}^{h}\right), b_{\lambda \mu} \dot{e}_{3}^{h}\right) \mathrm{d} \tau \leq C \int_{0}^{t}\left[\left|\dot{e}_{3}^{h}\right|_{0, \Omega}^{2}+\left|\gamma_{\alpha \beta}-\tilde{\gamma}_{\alpha \beta}^{h}\right|_{0, \Omega}^{2}\right] \mathrm{d} \tau
$$

Combining the estimates in Steps 1-4 provides the result stated in the proposition.

Notice that the constants in Propositions 3.1-3.4 are generic and do not depend on $h$ or $\gamma$. 


\subsubsection{Completion of the Proof of the Lemma 3.2}

We are now in a position to compute the final estimates of the error. Applying Propositions 3.1-3.4, and the coercivity property of the tensor of elastic modulus, $E^{\alpha \beta \lambda \mu}$, to the error equation (2.14), leads to the inequality

$$
\begin{aligned}
& \left|\dot{e}_{\alpha}^{h}(t)\right|_{0, \Omega}^{2}+\left|\dot{e}_{3}^{h}(t)\right|_{0, \Omega}^{2}+\gamma\left|\dot{e}_{3}^{h}(t)\right|_{1, \Omega}^{2}+\left|e_{3}^{h}(t)\right|_{2, \Omega}^{2}+\left|\left(\gamma_{\alpha \beta}^{h}-\tilde{\gamma}_{\alpha \beta}^{h}\right)(t)\right|_{0, \Omega}^{2} \\
& \leq \epsilon \sup _{0 \leq \tau \leq t}\left|e_{\alpha}^{h}(\tau)\right|_{1, \Omega}^{2}+C \int_{0}^{t}\left|\dot{e}_{\alpha}^{h}(\tau)\right|_{0, \Omega}^{2} \mathrm{~d} \tau+\epsilon \sup _{0 \leq \tau \leq t}\left|e_{3}^{h}(\tau)\right|_{2, \Omega}^{2} \\
& +\int_{0}^{t} C_{T, \epsilon} \sup _{0 \leq \tau \leq t}\left|\tilde{\gamma}_{\alpha \beta}^{h}(\tau)\right|_{0, \Omega}^{2}\left[\left|\dot{e}_{3}^{h}(\tau)\right|_{0, \Omega}^{2}+\gamma\left|\dot{e}_{3}^{h}(\tau)\right|_{1, \Omega}^{2}\right] \mathrm{d} \tau+C_{\epsilon_{0}} \sup _{0 \leq \tau \leq t}\left|\dot{\tilde{\gamma}}_{\alpha, \beta}^{h}(\tau)\right|_{L_{1+\epsilon_{0}}(\Omega)} \int_{0}^{t}\left|e_{3}^{h}(\tau)\right|_{2, \Omega}^{2} \mathrm{~d} \tau \\
& +C_{\epsilon} \sup _{0 \leq \tau \leq t}\left|\dot{\tilde{u}}_{3}^{h}\right|_{W^{1,2+\epsilon}(\Omega)}\left[\int_{0}^{t}\left|e_{3}^{h}(\tau)\right|_{2, \Omega}^{2} \mathrm{~d} \tau+\int_{0}^{t}\left|\left(\gamma_{\alpha \beta}^{h}-\tilde{\gamma}_{\alpha \beta}^{h}\right)(\tau)\right|_{0, \Omega}^{2} \mathrm{~d} \tau\right]+C_{T, \epsilon} Y(t) . \quad(3.1
\end{aligned}
$$

Additionally, we make use of the fact that

$$
\gamma_{\alpha \beta}^{h}-\tilde{\gamma}_{\alpha \beta}^{h}=\epsilon_{\alpha \beta}\left(\mathbf{e}^{h}\right)-b_{\alpha \beta} e_{3}^{h}+\frac{1}{2}\left(e_{3, \alpha}^{h} u_{3, \beta}^{h}+\tilde{u}_{3, \alpha}^{h} e_{3, \beta}^{h}\right)
$$

and take advantage of Korn's inequality (2.4) for shells to obtain a coercivity property for the error term given by

$$
\left|e_{\alpha}^{h}\right|_{1, \Omega}^{2} \leq C\left[\left|\gamma_{\alpha \beta}^{h}-\tilde{\gamma}_{\alpha \beta}^{h}\right|_{0, \Omega}^{2}+\left|e_{3}^{h}\right|_{2, \Omega}^{2}\right]\left[1+C_{0}\left|u_{3}^{h}\right|_{W^{1,4}(\Omega)}\left|\tilde{u}_{3}^{h}\right|_{W^{1,4}(\Omega)}\right] .
$$

Thus, combining (3.18) with (3.17) yields

$$
\begin{aligned}
\left|\dot{e}_{\alpha}^{h}(t)\right|_{0, \Omega}^{2}+\left|\dot{e}_{3}^{h}(t)\right|_{0, \Omega}^{2}+ & \gamma\left|\dot{e}_{3}^{h}(t)\right|_{1, \Omega}^{2}+\left|e_{3}^{h}(t)\right|_{2, \Omega}^{2}+\left|\left(\gamma_{\alpha \beta}^{h}-\tilde{\gamma}_{\alpha \beta}^{h}\right)(t)\right|_{0, \Omega}^{2}+\left|e_{\alpha}^{h}(t)\right|_{1, \Omega}^{2} \\
\leq & C\left[1+C_{0} \sup _{0 \leq \tau \leq t}\left|u_{3}^{h}(\tau)\right|_{W^{1,4}(\Omega)}\left|\tilde{u}_{3}^{h}(\tau)\right|_{W^{1,4}(\Omega)}\right]\left\{\epsilon \sup _{0 \leq \tau \leq t}\left[\left|e_{\alpha}^{h}(\tau)\right|_{1, \Omega}^{2}+\left|e_{3}^{h}(\tau)\right|_{2, \Omega}^{2}\right]\right. \\
& +C_{T, \epsilon} \sup _{0 \leq \tau \leq t}\left[\left|\tilde{\gamma}_{\alpha \beta}^{h}(\tau)\right|_{0, \Omega}^{2}+\left|\dot{\tilde{\gamma}}_{\alpha \beta}^{h}(\tau)\right|_{L_{1+\epsilon_{0}}(\Omega)}+\left|\dot{\tilde{u}}_{3}^{h}(\tau)\right|_{W^{1,2+\epsilon}(\Omega)}\right] \\
& \left.\times \int_{0}^{t}\left[\left|e_{3}^{h}(\tau)\right|_{2, \Omega}^{2}+\left|\dot{e}_{\alpha}^{h}(\tau)\right|_{0, \Omega}^{2}+\gamma\left|\dot{e}_{3}^{h}(\tau)\right|_{1, \Omega}^{2}+\left|\left(\gamma_{\alpha \beta}^{h}-\tilde{\gamma}_{\alpha \beta}^{h}\right)(\tau)\right|_{0, \Omega}^{2}\right] \mathrm{d} \tau+C_{T, \epsilon} Y(t)\right\}
\end{aligned}
$$

This estimate is further simplified by taking $\epsilon$ to be suitably small and applying Gronwall's inequality to obtain

$$
\begin{aligned}
& \left|\dot{e}_{\alpha}^{h}(t)\right|_{0, \Omega}^{2}+\left|\dot{e}_{3}^{h}(t)\right|_{0, \Omega}^{2}+\gamma\left|\dot{e}_{3}^{h}(t)\right|_{1, \Omega}^{2}+\left|e_{3}^{h}(t)\right|_{2, \Omega}^{2}+\left|e_{\alpha}^{h}(t)\right|_{1, \Omega}^{2} \\
& \quad \leq C_{T} \sup _{0 \leq \tau \leq t}\left[\left|u_{3}^{h}(\tau)\right|_{W^{1,4}(\Omega)}\left|\tilde{u}_{3}^{h}(\tau)\right|_{W^{1,4}(\Omega)}\left(\left|\tilde{\gamma}_{\alpha \beta}^{h}(\tau)\right|_{0, \Omega}^{2}+\left|\dot{\tilde{\gamma}}_{\alpha \beta}^{h}(\tau)\right|_{L_{1+\epsilon_{0}}(\Omega)}+\left|\dot{\tilde{u}}_{3}^{h}(\tau)\right|_{W^{1,2+\epsilon}(\Omega)}\right)\right] Y(t)
\end{aligned}
$$

where $C_{T}$ is independent of $\gamma>0$.

Recalling the stability result of Lemma 2.2 and using Sobolev embeddings, we obtain

$$
\left|u_{3}^{h}(t)\right|_{W^{1,4}(\Omega)}^{2} \leq C\left[E_{\gamma}^{h}(0)+|(\vec{p}, q)|^{2}\right] \leq C\left(E_{\gamma}(0),|(\vec{p}, q)|\right) \text { for all } t
$$

Moreover, recalling the embedding $W^{1,4}(\Omega) \supset H^{2}(\Omega)$ and stability properties of interpolants in higher norms (see (1.4)) imply

$$
\begin{gathered}
\left|\tilde{u}_{3}^{h}(t)\right|_{W^{1,4}(\Omega)}^{2} \leq\left|u t_{3}^{h}(t)\right|_{2, \Omega}^{2} \leq C\left|u_{3}(t)\right|_{2, \Omega}^{2} \leq C\left(E_{\gamma}(0),|\vec{p}, q|\right) \\
\left|\dot{\tilde{u}}_{3}^{h}(t)\right|_{W^{1,2+\epsilon}(\Omega)} \leq\left|\dot{\tilde{u}}_{3}^{h}(t)\right|_{1+\frac{\epsilon}{2+\epsilon}, \Omega} \leq C\left|\dot{u}_{3}(t)\right|_{1+\epsilon_{0}, \Omega} \\
\left|\tilde{\gamma}_{\alpha \beta}^{h}(t)\right|_{0, \Omega}^{2} \leq C\left[\left|\tilde{u}_{\alpha}^{h}(t)\right|_{1, \Omega}^{2}+\left|\tilde{u}_{3}^{h}(t)\right|_{2, \Omega}^{4}\right] \leq C\left[\left|u_{\alpha}(t)\right|_{1, \Omega}^{2}+\left|u_{3}(t)\right|_{2, \Omega}^{4}\right] \leq C\left(E_{\gamma}(0),|\vec{p}, q|\right),
\end{gathered}
$$


where we have used $H^{2-\frac{2}{2+\epsilon}}(\Omega) \subset W^{1,2+\epsilon}(\Omega)$. The results stated in (3.20-3.23) applied to equation (3.19) provide the estimate stated in the Lemma 3.2 .

\subsection{The error for the interpolants}

It remains is to provide estimates for the " $Y$ " term, which represents the error resulting from approximations of the original solution. This will be done, as usual, by using the approximation error of the associated Sobolev spaces.

Lemma 3.3. For all $t \leq T$

$$
\begin{aligned}
Y(t) \leq & C_{T, \epsilon}\left(E_{\gamma}(0),|(\vec{p}, q)|,\left|\dot{u}_{\alpha}\right|_{\epsilon, \infty},\left|\dot{u}_{3}\right|_{1+\epsilon, \infty}\right)\left(\int_{0}^{t}\left[h^{2(s-1)}\left(\left|\dot{u}_{\alpha}\right|_{s, \Omega}^{2}+\left|u_{\alpha}\right|_{s, \Omega}^{2}\right)+h^{2(r-2)}\left(\left|\dot{u}_{3}\right|_{r, \Omega}^{2}+\left|u_{3}\right|_{r, \Omega}^{2}\right)\right] \mathrm{d} \tau\right) \\
& +C \int_{0}^{t} h^{2(r-2)}\left|\dot{u}_{3}\right|_{r, \Omega}^{2} \mathrm{~d} \tau+C \int_{0}^{t}\left[h^{2(s-1)}\left|\ddot{u}_{\alpha}\right|_{s-1, \Omega}^{2}+h^{2(r-2)}\left|\ddot{u}_{3}\right|_{r-2, \Omega}^{2}+\gamma h^{2(r-2)}\left|\ddot{u}_{3}\right|_{r-1, \Omega}^{2}\right] \mathrm{d} \tau . \quad(3.24)
\end{aligned}
$$

Proof of Lemma 3.3. The following inequality, following from Sobolev's embeddings and Cauchy-Shwarz inequality, will be used frequently.

$$
|f g|_{0, \Omega} \leq C|f|_{1, \Omega}|g|_{\epsilon, \Omega} \forall \epsilon>0 .
$$

Recall $Y(t)=Y_{u}(t)+Y_{0}$ where

$$
\begin{aligned}
Y_{u}(t) \equiv & \left(\int_{0}^{t}\left|\dot{\gamma}_{\alpha \beta}-\dot{\tilde{\gamma}}_{\alpha \beta}^{h}\right|_{0, \Omega} \mathrm{d} \tau\right)^{2}+\int_{0}^{t}\left[\left|\ddot{u}_{\alpha}-\ddot{\tilde{u}}_{\alpha}^{h}\right|_{0, \Omega}^{2}+\left|\ddot{u}_{3}-\ddot{\tilde{u}}_{3}^{h}\right|_{0, \Omega}^{2}+\gamma\left|\ddot{u}_{3}-\ddot{\tilde{u}}_{3}^{h}\right|_{1, \Omega}^{2}+\left|\gamma_{\alpha \beta}-\tilde{\gamma}_{\alpha \beta}^{h}\right|_{0, \Omega}^{2}\right] \mathrm{d} \tau \\
& \times\left(\int_{0}^{t}\left|\dot{u}_{3}-\dot{\tilde{u}}_{3}^{h}\right|_{2, \Omega} \mathrm{d} \tau\right)^{2}+\left(\int_{0}^{t}\left|\frac{\mathrm{d}}{\mathrm{d} t}\left(\tilde{\gamma}_{\alpha \beta}^{h} \tilde{u}_{3, \lambda}^{h}-\gamma_{\alpha \beta} u_{3, \lambda}\right)\right|_{-1, \Omega} \mathrm{d} \tau\right)^{2}
\end{aligned}
$$

Proposition stated below provides the estimate for $Y_{u}$ term.

\section{Proposition 3.5.}

$$
\begin{gathered}
Y_{u}(t) \leq C_{T, \epsilon}\left(E_{\gamma}(0),|(\vec{p}, q)|,\left|\dot{u}_{\alpha}\right|_{\epsilon, \infty},\left|\dot{u}_{3}\right|_{1+\epsilon, \infty}\right)\left(\int _ { 0 } ^ { t } \left[h^{2(s-1)}\left(\left|\dot{u}_{\alpha}\right|_{s, \Omega}^{2}+\left|u_{\alpha}\right|_{s, \Omega}^{2}\right)\right.\right. \\
\left.\left.+h^{2(r-2)}\left(\left|\dot{u}_{3}\right|_{r, \Omega}^{2}+\left|u_{3}\right|_{r, \Omega}^{2}\right)\right] \mathrm{d} \tau\right)+C_{\epsilon} \int_{0}^{t} h^{2(r-2)}\left|\dot{u}_{3}\right|_{r, \Omega}^{2} \mathrm{~d} \tau \\
\quad+C_{\epsilon} \int_{0}^{t}\left[h^{2(s-1)}\left|\ddot{u}_{\alpha}\right|_{s-1, \Omega}^{2}+h^{2(r-2)}\left|\ddot{u}_{3}\right|_{r-2, \Omega}^{2}+\gamma h^{2(r-2)}\left|\ddot{u}_{3}\right|_{r-1, \Omega}^{2}\right] \mathrm{d} \tau
\end{gathered}
$$

Proof. The most involved parts to estimate will be the first and last terms on the right-hand side of (3.26).

Step 1.

We begin by using the definition of the middle surface strain tensor, $\gamma_{\alpha \beta}$, and note the relationship

$$
\begin{aligned}
\dot{\gamma}_{\alpha \beta}-\dot{\tilde{\gamma}}_{\alpha \beta}^{h}= & \epsilon_{\alpha \beta}\left(\dot{\vec{u}}-\dot{\overrightarrow{\tilde{u}}}^{h}\right)-b_{\alpha \beta}\left(\dot{u}_{3}-\dot{\tilde{u}}_{3}^{h}\right)+\frac{1}{2}\left(\dot{u}_{3, \alpha} u_{3, \beta}+u_{3, \alpha} \dot{u}_{3, \beta}\right)-\frac{1}{2}\left(\dot{\tilde{u}}_{3, \alpha}^{h} \tilde{u}_{3, \beta}^{h}+\tilde{u}_{3, \alpha}^{h} \dot{\tilde{u}}_{3, \beta}^{h}\right) \\
= & \epsilon_{\alpha \beta}\left(\dot{\vec{u}}-\dot{\vec{u}}^{h}\right)-b_{\alpha \beta}\left(\dot{u}_{3}-\dot{\tilde{u}}_{3}^{h}\right)+\frac{1}{2}\left[\left(\dot{u}_{3, \alpha}-\dot{\tilde{u}}_{3, \alpha}^{h}\right) \tilde{u}_{3, \beta}^{h}\right. \\
& \left.+\dot{u}_{3, \alpha}\left(u_{3, \beta}-\tilde{u}_{3, \beta}^{h}\right)+\left(\dot{u}_{3, \beta}-\dot{\tilde{u}}_{3, \beta}^{h}\right) \tilde{u}_{3, \alpha}^{h}+\dot{u}_{3, \beta}\left(u_{3, \alpha}-\tilde{u}_{3, \alpha}^{h}\right)\right] .
\end{aligned}
$$


The estimates for the linear terms are straightforward. We shall concentrate on the nonlinear terms. In particular, using (3.25), (1.4) and stability estimates for interpolants we note that

$$
\begin{aligned}
\left|\left(\dot{u}_{3, \alpha}-\dot{\tilde{u}}_{3, \alpha}^{h}\right) \tilde{u}_{3, \beta}^{h}\right|_{0, \Omega} & \leq C\left|\dot{u}_{3}-\dot{\tilde{u}}_{3}^{h}\right|_{2, \Omega}\left|\tilde{u}_{3, \beta}^{h}\right|_{\epsilon, \Omega} \leq C h^{r-2}\left|\dot{u}_{3}\right|_{r, \Omega}\left|\tilde{u}_{3}^{h}\right|_{1+\epsilon, \Omega} \\
& \leq C h^{r-2}\left|\dot{u}_{3}\right|_{r, \Omega}\left|u_{3}\right|_{1+\epsilon, \Omega} \leq C\left(E_{\gamma}(0),|\vec{p}, q|\right) h^{r-2}\left|\dot{u}_{3}\right|_{r, \Omega} .
\end{aligned}
$$

Similarly,

$$
\left|\dot{u}_{3, \alpha}\left(u_{3, \beta}-\tilde{u}_{3, \beta}^{h}\right)\right|_{0, \Omega} \leq C_{\epsilon}\left|u_{3, \beta}-\tilde{u}_{3, \beta}^{h}\right|_{1, \Omega}\left|\dot{u}_{3, \alpha}\right|_{\epsilon, \Omega} \leq C_{\epsilon}\left|u_{3}-\tilde{u}_{3}^{h}\right|_{2, \Omega}\left|\dot{u}_{3}\right|_{1+\epsilon, \Omega} \leq C_{\epsilon} h^{r-2}\left|u_{3}\right|_{r, \Omega}\left|\dot{u}_{3}\right|_{1+\epsilon, \Omega}
$$

Thus, from (3.28), the estimates in $(3.29,3.30)$ imply

$$
\begin{aligned}
\left|\dot{\gamma}_{\alpha \beta}-\dot{\tilde{\gamma}}_{\alpha \beta}^{h}\right|_{0, \Omega} & \left.\leq C\left(E_{\gamma}(0),|(\vec{p}, q)|\right)\right)\left[h^{s-1}\left|\dot{u}_{\alpha}\right|_{s, \Omega}+h^{r-1}\left|\dot{u}_{3}\right|_{r-1, \Omega}+h^{r-2}\left|\dot{u}_{3}\right|_{r, \Omega}+h^{r-2}\left|u_{3}\right|_{r, \Omega}\left|\dot{u}_{3}\right|_{1+\epsilon, \Omega}\right] \\
& \leq C_{\epsilon}\left(E_{\gamma}(0),|(\vec{p}, q)|,\left|\dot{u}_{3}\right|_{1+\epsilon, \Omega}\right)\left[h^{s-1}\left|\dot{u}_{\alpha}\right|_{s, \Omega}+h^{r-2}\left|\dot{u}_{3}\right|_{r, \Omega}\right] .
\end{aligned}
$$

\section{Step 2.}

Since

$$
\gamma_{\alpha \beta}-\tilde{\gamma}_{\alpha \beta}^{h}=\epsilon_{\alpha \beta}\left(\vec{u}-\overrightarrow{\tilde{u}}^{h}\right)-b_{\alpha \beta}\left(u_{3}-\tilde{u}_{3}^{h}\right)+\frac{1}{2}\left[\left(u_{3, \alpha}-\tilde{u}_{3, \alpha}^{h}\right) \tilde{u}_{3, \beta}^{h}+u_{3, \alpha}\left(u_{3, \beta}-\tilde{u}_{3, \beta}^{h}\right)\right]
$$

and $(3.25,1.4)$ imply that

$$
\begin{aligned}
\left|\left(u_{3, \alpha}-\tilde{u}_{3, \alpha}^{h}\right) \tilde{u}_{3, \beta}^{h}\right|_{0, \Omega} & \leq C\left|u_{3, \alpha}-\tilde{u}_{3, \alpha}^{h}\right|_{1, \Omega}\left|\tilde{u}_{3, \beta}^{h}\right|_{\epsilon, \Omega} \leq C\left|u_{3}-\tilde{u}_{3}^{h}\right|_{2, \Omega}\left|\tilde{u}_{3}^{h}\right|_{1+\epsilon, \Omega} \\
& \leq C h^{r-2}\left|u_{3}\right|_{r, \Omega}\left|u_{3}\right|_{1+\epsilon, \Omega} \leq C\left(E_{\gamma}(0),|\vec{p}, q|\right) h^{r-2}\left|u_{3}\right|_{r, \Omega} .
\end{aligned}
$$

and

$$
\left|u_{3, \beta}-\tilde{u}_{3, \beta}^{h}\right|_{0, \Omega} \leq C h^{r-1}\left|u_{3}\right|_{r, \Omega},
$$

we obtain

$$
\begin{aligned}
\left|\gamma_{\alpha \beta}-\tilde{\gamma}_{\alpha \beta}^{h}\right|_{0, \Omega} & \leq C\left(E_{\gamma}(0),|\vec{p}, q|\right)\left[h^{s-1}\left|u_{\alpha}\right|_{s, \Omega}+h^{r-1}\left|u_{3}\right|_{r, \Omega}+h^{r-2}\left|u_{3}\right|_{r, \Omega}+h^{r}\left|u_{3}\right|_{r, \Omega}\right] \\
& \leq C\left(E_{\gamma}(0),|\vec{p}, q|\right)\left[h^{s-1}\left|u_{\alpha}\right|_{s, \Omega}+h^{r-2}\left|u_{3}\right|_{r, \Omega}\right] .
\end{aligned}
$$

\section{Step 3.}

In order to estimate the last term in (3.26), we will need the following estimate for negative norms.

Proposition 3.6. Under the regularity assumptions associated with the approximations specified in (1.4), we have

$$
\left|\frac{\mathrm{d}}{\mathrm{d} t}\left(\tilde{\gamma}_{\alpha \beta}^{h} \tilde{u}_{3, \lambda}^{h}-\gamma_{\alpha \beta} u_{3, \lambda}\right)\right|_{-1, \Omega} \leq C_{\epsilon}\left(E_{\gamma}(0),|\vec{p}, q|,\left|\dot{u}_{3}\right|_{1+\epsilon, \Omega},\left|\dot{u}_{\alpha}\right|_{\epsilon, \Omega}\right)\left[h^{s-1}\left|\dot{u}_{\alpha}\right|_{s, \Omega}+h^{r-2}\left[\left|\dot{u}_{3}\right|_{r, \Omega}+\left|u_{3}\right|_{r, \Omega}\right]\right] .
$$

Proof. First, we observe

$$
\frac{\mathrm{d}}{\mathrm{d} t}\left(\tilde{\gamma}_{\alpha \beta}^{h} \tilde{u}_{3, \lambda}^{h}-\gamma_{\alpha \beta} u_{3, \lambda}\right)=\left(\dot{\tilde{\gamma}}_{\alpha \beta}^{h}-\dot{\gamma}_{\alpha \beta}\right) \tilde{u}_{3, \lambda}^{h}+\dot{\gamma}_{\alpha \beta}\left(\tilde{u}_{3, \lambda}^{h}-u_{3, \lambda}\right)+\left(\tilde{\gamma}_{\alpha \beta}^{h}-\gamma_{\alpha \beta}\right) \dot{\tilde{u}}_{3, \lambda}^{h}+\gamma_{\alpha \beta}\left(\dot{\tilde{u}}_{3, \lambda}^{h}-\dot{u}_{3, \lambda}\right) .
$$


Then, using the definition of the negative norm and the Cauchy-Schwarz and Holder's inequalities, we have the estimate

$$
|f g|_{-1, \Omega}=\sup _{\phi \in H^{1}(\Omega)} \frac{\left|\int_{\Omega} f g \phi \mathrm{d} x\right|}{|\phi|_{1, \Omega}} \leq \frac{|f|_{0, \Omega}|g|_{L_{2 \bar{p}}(\Omega)}|\phi|_{L_{2 p}(\Omega)}}{|\phi|_{1, \Omega}} \text { with } \frac{1}{p}+\frac{1}{\bar{p}}=1 .
$$

This result can be simplified by applying the Sobolev embeddings

$$
H^{1}(\Omega) \subset L_{2 p}(\Omega) \text { for any } p<\infty
$$

and taking $p=\frac{2+\epsilon_{1}}{\epsilon_{1}}$ and $2 \bar{p}=2+\epsilon_{1}$, and

$$
H^{\epsilon_{0}}(\Omega) \subset L_{2+\epsilon_{1}}(\Omega) \text { with } \epsilon_{0}=\frac{\epsilon_{1}}{2+\epsilon_{1}}
$$

to obtain

$$
\frac{|f|_{0, \Omega}|g|_{L_{\bar{p}}(\Omega)}|\phi|_{L_{p}(\Omega)}}{|\phi|_{1, \Omega}} \leq C_{\epsilon_{0}}|f|_{0, \Omega}|g|_{\epsilon_{0}, \Omega}
$$

Hence,

$$
|f g|_{-1, \Omega} \leq C_{\epsilon_{0}}|f|_{0, \Omega}|g|_{\epsilon_{0}, \Omega} \text { for any } \epsilon_{0}>0 .
$$

Similarly, Green's formula can be used to show

$$
\left|\epsilon_{\alpha \beta}(f) g\right|_{-1, \Omega} \leq C_{\epsilon_{0}}|f|_{0, \Omega}|g|_{1+\epsilon_{0}, \Omega}
$$

Another estimate we need is determined by using interpolation theory of Besov spaces [21] which provides us with

$$
|f g|_{\epsilon, \Omega} \leq C|f|_{1-\epsilon_{0}, \Omega}|g|_{\epsilon+\epsilon_{0}, \Omega} \forall \epsilon, \epsilon_{0}>0 \text { and } \epsilon+\epsilon_{0}<1 .
$$

From here, it follows, by Holder's inequality and duality,

$$
|f g|_{-1, \Omega} \leq C_{\epsilon}|f|_{-1+\epsilon, \Omega}|g|_{1-\epsilon / 2, \Omega} \forall \epsilon>0 .
$$

The remainder of the proof relies on applying (3.37-3.39) to appropriate terms involving the right-hand side of (3.36).

First, we decompose $\dot{\tilde{\gamma}}_{\alpha \beta}^{h}-\dot{\gamma}_{\alpha \beta}$ in a similar manner as in (3.28) to obtain the estimate for the first term on the right-hand side of (3.36). The details are provided below.

First notice that by applying (3.39) and (1.4), we obtain

$$
\begin{aligned}
\left|\epsilon_{\alpha \beta}\left(\dot{\overrightarrow{\tilde{u}}}^{h}-\dot{\vec{u}}\right) \tilde{u}_{3, \lambda}^{h}\right|_{-1, \Omega} & \leq C\left|\epsilon_{\alpha \beta}\left(\dot{\overrightarrow{\tilde{u}}}^{h}-\dot{\vec{u}}\right)\right|_{-1+\epsilon, \Omega}\left|\tilde{u}_{3, \lambda}^{h}\right|_{1, \Omega} \leq C\left|\dot{\tilde{u}}_{\alpha}^{h}-\dot{u}_{\alpha}\right|_{\epsilon, \Omega}\left|\tilde{u}_{3}^{h}\right|_{2, \Omega} \\
& \leq C h^{s-\epsilon}\left|\dot{u}_{\alpha}\right|_{s, \Omega}\left|u_{3}\right|_{2, \Omega} \leq C\left(E_{\gamma}(0),|(\vec{p}, q)|\right) h^{s-1}\left|\dot{u}_{\alpha}\right|_{s, \Omega} .
\end{aligned}
$$

Similarly, (1.4, 3.36, 3.37, 3.39) can be used to show

$$
\begin{aligned}
\left|b_{\alpha \beta}\left(\dot{\tilde{u}}_{3}^{h}-\dot{u}_{3}\right) \tilde{u}_{3, \lambda}^{h}\right|_{-1, \Omega} & \leq C\left|\dot{\tilde{u}}_{3}^{h}-\dot{u}_{3}\right|_{0, \Omega}\left|\tilde{u}_{3, \lambda}^{h}\right|_{\epsilon_{0}, \Omega} \leq C h^{r-1}\left|\dot{u}_{3}\right|_{r-1, \Omega}\left|u_{3}\right|_{1+\epsilon_{0}, \Omega} \\
& \leq C\left(E_{\gamma}(0),|(\vec{p}, q)|\right) h^{r-1}\left|\dot{u}_{3}\right|_{r-1, \Omega},
\end{aligned}
$$




$$
\begin{aligned}
\left|\left(\dot{\tilde{u}}_{3, \alpha}^{h}-\dot{u}_{3, \alpha}\right) \tilde{u}_{3, \beta}^{h} \tilde{u}_{3, \lambda}^{h}\right|_{-1, \Omega} & \leq C\left|\dot{\tilde{u}}_{3, \alpha}^{h}-\dot{u}_{3, \alpha}\right|_{\epsilon, \Omega}\left|\tilde{u}_{3, \beta}^{h} \tilde{u}_{3, \lambda}^{h}\right|_{0, \Omega} \leq C h^{r-1-\epsilon}\left|\dot{u}_{3}\right|_{r, \Omega}\left|\tilde{u}_{3, \beta}^{h}\right|_{1, \Omega}\left|\tilde{u}_{3, \lambda}^{h}\right|_{\epsilon, \Omega} \\
& \leq C h^{r-1-\epsilon}\left|\dot{u}_{3}\right|_{r, \Omega}\left|u_{3}\right|_{2, \Omega}\left|u_{3}\right|_{1+\epsilon, \Omega} \leq C\left(E_{\gamma}(0),|(\vec{p}, q)|\right) h^{r-1-\epsilon}\left|\dot{u}_{3}\right|_{r, \Omega},
\end{aligned}
$$

and

$$
\begin{aligned}
\left|\dot{u}_{3, \alpha}\left(\tilde{u}_{3, \beta}^{h}-u_{3, \beta}\right) \tilde{u}_{3, \lambda}^{h}\right|_{-1, \Omega} & \leq C_{\epsilon}\left|\tilde{u}_{3, \beta}^{h}-u_{3, \beta}\right|_{\epsilon, \Omega}\left|\dot{u}_{3, \alpha} \tilde{u}_{3, \lambda}^{h}\right|_{0, \Omega} \leq C h^{r-1-\epsilon}\left|u_{3}\right|_{r, \Omega}\left|\tilde{u}_{3, \lambda}^{h}\right|_{1, \Omega}\left|\dot{u}_{3, \alpha}\right|_{\epsilon, \Omega} \\
& \leq C_{\epsilon} h^{r-1-\epsilon}\left|u_{3}\right|_{r, \Omega}\left|\tilde{u}_{3}^{h}\right|_{2, \Omega}\left|\dot{u}_{3}\right|_{1+\epsilon, \Omega} \leq C_{\epsilon} h^{r-1-\epsilon}\left|u_{3}\right|_{r, \Omega}\left|u_{3}\right|_{2, \Omega}\left|\dot{u}_{3}\right|_{1+\epsilon, \Omega} \\
& \leq C_{\epsilon}\left(E_{\gamma}(0),|(\vec{p}, q)|,\left|\dot{u}_{3}\right|_{1+\epsilon, \Omega}\right) h^{r-2}\left|u_{3}\right|_{r, \Omega}
\end{aligned}
$$

Thus, by combining (3.40-3.43) we have

$$
\begin{aligned}
\left|\left(\dot{\tilde{\gamma}}_{\alpha \beta}^{h}-\dot{\gamma}_{\alpha \beta}\right) \tilde{u}_{3, \lambda}^{h}\right|_{-1, \Omega} & \leq C_{\epsilon}\left(E_{\gamma}(0),|(\vec{p}, q)|,\left|\dot{u}_{3}\right|_{1+\epsilon, \Omega}\right)\left[h^{s-1}\left|\dot{u}_{\alpha}\right|_{s, \Omega}+h^{r-1}\left|\dot{u}_{3}\right|_{r-1, \Omega}+h^{r-2}\left(\left|\dot{u}_{3}\right|_{r, \Omega}+\left|u_{3}\right|_{r, \Omega}\right)\right] \\
& \leq C_{\epsilon}\left(E_{\gamma}(0),|(\vec{p}, q)|,\left|\dot{u}_{3}\right|_{1+\epsilon, \Omega}\right)\left[h^{s-1}\left|\dot{u}_{\alpha}\right|_{s, \Omega}+h^{r-2}\left(\left|\dot{u}_{3}\right|_{r, \Omega}+\left|u_{3}\right|_{r, \Omega}\right)\right] .
\end{aligned}
$$

Similarly,

$$
\begin{aligned}
\left|\left(\tilde{\gamma}_{\alpha \beta}^{h}-\gamma_{\alpha \beta}\right) \dot{\tilde{u}}_{3, \lambda}^{h}\right|_{-1, \Omega} \leq & C_{\epsilon}\left[\left|\epsilon_{\alpha \beta}\left(\overrightarrow{\tilde{u}}^{h}-\vec{u}\right)\right|_{0, \Omega}\left|\dot{\tilde{u}}_{3, \lambda}^{h}\right|_{\epsilon, \Omega}+\left|\tilde{u}_{3}^{h}-u_{3}\right|_{0, \Omega}\left|\dot{\tilde{u}}_{3, \lambda}^{h}\right|_{\epsilon, \Omega}\right. \\
& \left.+\left|\tilde{u}_{3, \alpha}^{h}-u_{3, \alpha}\right|_{1, \Omega}\left|\tilde{u}_{3, \beta}^{h} \dot{\tilde{u}}_{3, \lambda}^{h}\right|_{-1+\epsilon, \Omega}\right] \\
\leq & C_{\epsilon} h^{s-1}\left|u_{\alpha}\right|_{s, \Omega}\left|\dot{u}_{3}\right|_{1+\epsilon, \Omega}+C_{\epsilon} h^{r-1}\left|u_{3}\right|_{r, \Omega}\left|\dot{u}_{3}\right|_{1+\epsilon, \Omega}+C_{\epsilon} h^{r-2}\left|u_{3}\right|_{r, \Omega}\left|u_{3}\right|_{2, \Omega}\left|\dot{u}_{3}\right|_{1+\epsilon, \Omega} \\
\leq & C_{\epsilon}\left(E_{\gamma}(0),||(\vec{p}, q)\left|, \dot{u}_{3}\right|_{1+\epsilon, \Omega}\right)\left[h^{s-1}\left|u_{\alpha}\right|_{s, \Omega}+h^{r-2}\left|u_{3}\right|_{r, \Omega}\right] .
\end{aligned}
$$

For the last two terms in (3.36) we have

$$
\left|\dot{\gamma}_{\alpha \beta}\left(\tilde{u}_{3, \lambda}^{h}-u_{3, \lambda}\right)\right|_{-1, \Omega} \leq C_{\epsilon}\left|\dot{\gamma}_{\alpha \beta}\right|_{-1+\epsilon, \Omega}\left|\tilde{u}_{3, \lambda}^{h}-u_{3, \lambda}\right|_{1, \Omega} \leq C_{\epsilon} h^{r-2}\left|u_{3}\right|_{r, \Omega}\left|\dot{\gamma}_{\alpha \beta}\right|_{-1+\epsilon, \Omega}
$$

but

$$
\begin{aligned}
\left|\dot{\gamma}_{\alpha \beta}\right|_{-1+\epsilon, \Omega} & \leq C_{\epsilon}\left[\left|\epsilon_{\alpha \beta}(\dot{\vec{u}})\right|_{-1+\epsilon, \Omega}+\left|\dot{u}_{3}\right|_{-1+\epsilon, \Omega}+\left|\dot{u}_{3, \alpha} u_{3, \beta}\right|_{-1+\epsilon, \Omega}\right] \leq C_{\epsilon}\left[\left|\dot{u}_{\alpha}\right|_{\epsilon, \Omega}+\left|\dot{u}_{3}\right|_{0, \Omega}+\left|\dot{u}_{3}\right|_{1+\epsilon, \Omega}\left|u_{3}\right|_{2, \Omega}\right] \\
& \leq C_{\epsilon}\left(E_{\gamma}(0),|(\vec{p}, q)|\right)\left[\left|\dot{u}_{\alpha}\right|_{\epsilon, \Omega}+\left|\dot{u}_{3}\right|_{1+\epsilon, \Omega}\right.
\end{aligned}
$$

Hence,

$$
\left|\dot{\gamma}_{\alpha \beta}\left(\tilde{u}_{3, \lambda}^{h}-u_{3, \lambda}\right)\right|_{-1, \Omega} \leq C_{\epsilon}\left(E_{\gamma}(0),|(\vec{p}, q)|,\left|\dot{u}_{\alpha}\right|_{\epsilon, \Omega},\left|\dot{u}_{3}\right|_{1+\epsilon, \Omega}\right) h^{r-2}\left|u_{3}\right|_{r, \Omega}
$$

and

$$
\begin{aligned}
\left|\gamma_{\alpha \beta}\left(\dot{\tilde{u}}_{3, \lambda}^{h}-\dot{u}_{3, \lambda}\right)\right|_{-1, \Omega} & \leq C_{\epsilon}\left|\gamma_{\alpha \beta}\right|_{0, \Omega}\left|\dot{\tilde{u}}_{3, \lambda}^{h}-\dot{u}_{3, \lambda}\right|_{\epsilon, \Omega} \leq C_{\epsilon}\left(E_{\gamma}(0),|(\vec{p}, q)|\right)\left|\dot{\tilde{u}}^{h}-\dot{u}_{3}\right|_{1+\epsilon, \Omega} \\
& \leq C_{\epsilon}\left(E_{\gamma}(0),|(\vec{p}, q)|\right) h^{r-1-\epsilon}\left|\dot{u}_{3}\right|_{r, \Omega} .
\end{aligned}
$$

Collecting $(3.44,3.47)$ provides the result in the Proposition 3.6. 


\section{Step 4.}

Now we are in a position to state the final estimate for $Y_{u}$. Indeed, by applying $(3.31,3.35)$, Proposition 3.6 and (1.4) to the definition of $Y_{u}$ in (3.26) we have

$$
\begin{aligned}
Y_{u} \leq C_{T, \epsilon} & \left(E_{\gamma}(0),|(\vec{p}, q)|,\left|\dot{u}_{\alpha}\right|_{\epsilon, \infty},\left|\dot{u}_{3}\right|_{1+\epsilon, \infty}\right)\left(\int_{0}^{t}\left[h^{2(s-1)}\left(\left|\dot{u}_{\alpha}\right|_{s, \Omega}^{2}+\left|u_{\alpha}\right|_{s, \Omega}^{2}\right)+h^{2(r-2)}\left(\left|\dot{u}_{3}\right|_{r, \Omega}^{2}+\left|u_{3}\right|_{r, \Omega}^{2}\right)\right] \mathrm{d} \tau\right) \\
& +C_{\epsilon} \int_{0}^{t} h^{2(r-2)}\left|\dot{u}_{3}\right|_{r, \Omega}^{2} \mathrm{~d} \tau+C_{\epsilon} \int_{0}^{t}\left[h^{2(s-1)}\left|\ddot{u}_{\alpha}\right|_{s-1, \Omega}^{2}+h^{2(r-2)}\left|\ddot{u}_{3}\right|_{r-2, \Omega}^{2}+\gamma h^{2(r-2)}\left|\ddot{u}_{3}\right|_{r-1, \Omega}^{2}\right] \mathrm{d} \tau \quad(3.48)
\end{aligned}
$$

which is the desired estimate in Proposition 3.5.

To complete the proof of Lemma it remains is to derive an estimate for $Y_{0}$. This is done in the proposition below.

\section{Proposition 3.7.}

$$
\begin{aligned}
Y_{0} & \leq C\left(E_{\gamma}(0),|(\vec{p}, q)|\right)\left[h^{2(s-1)}\left|u_{\alpha}(0)\right|_{s, \Omega}^{2}+h^{2(r-2)}\left|u_{3}(0)\right|_{r, \Omega}^{2}\right] \\
& \leq C\left(E_{\gamma}(0),|(\vec{p}, q)|\right)\left[h^{2(s-1)}\left[\left|u_{\alpha}\right|_{s, 2}^{2}+\left|\dot{u}_{\alpha}\right|_{s, 2}^{2}\right]+h^{2(r-2)}\left[\left|u_{3}\right|_{r, 2}^{2}+\left|\dot{u}_{3}\right|_{r, 2}^{2}\right]\right] .
\end{aligned}
$$

Proof. To this end, from (3.6) and Lemma 2.2 we have

$$
\begin{aligned}
Y_{0} \leq & C\left(E_{\gamma}(0),|(\vec{p}, q)|\right)\left[\left|e_{3}^{h}(0)\right|_{2, \Omega}^{2}+\mathcal{E}(0)+\left|e_{\alpha}^{h}(0)\right|_{1, \Omega}^{2}+\left|\gamma_{\alpha \beta}(0)-\tilde{\gamma}_{\alpha \beta}^{h}(0)\right|_{0, \Omega}^{2}+\left|u_{3}(0)-\tilde{u}_{3}^{h}(0)\right|_{2, \Omega}^{2}\right. \\
& \left.+\left|\gamma_{\alpha \beta}(0)\left(u_{3, \lambda}(0)-\tilde{u}_{3, \lambda}^{h}(0)\right)\right|_{-1, \Omega}^{2}+\left|\left(\tilde{\gamma}_{\alpha \beta}^{h}(0)-\gamma_{\alpha \beta}(0)\right) u_{3, \lambda}(0)\right|_{-1, \Omega}^{2}\right] .
\end{aligned}
$$

However, recalling (3.37) we have

$$
\left|\gamma_{\alpha \beta}(0)\left(u_{3, \lambda}(0)-\tilde{u}_{3, \lambda}^{h}(0)\right)\right|_{-1, \Omega}^{2} \leq\left|\gamma_{\alpha \beta}(0)\right|_{0, \Omega}^{2}\left|u_{3, \lambda}(0)-\tilde{u}_{3, \lambda}^{h}(0)\right|_{\epsilon, \Omega}^{2} \leq C_{\epsilon}\left(E_{\gamma}(0)\right) h^{2(r-1-\epsilon)}\left|u_{3}(0)\right|_{r, \Omega}^{2}
$$

and

$$
\left|\left(\tilde{\gamma}_{\alpha \beta}^{h}(0)-\gamma_{\alpha \beta}(0)\right) u_{3, \lambda}(0)\right|_{-1, \Omega}^{2} \leq C\left|\tilde{\gamma}_{\alpha \beta}^{h}(0)-\gamma_{\alpha \beta}(0)\right|_{0, \Omega}^{2}\left|u_{3, \lambda}(0)\right|_{\epsilon, \Omega}^{2} \leq C\left(E_{\gamma}(0)\right)\left|\tilde{\gamma}_{\alpha \beta}^{h}(0)-\gamma_{\alpha \beta}(0)\right|_{0, \Omega}^{2}
$$

Thus,

$$
\begin{aligned}
Y_{0} \leq & C\left(E_{\gamma}(0),|(\vec{p}, q)|\right)\left[\left|e_{3}^{h}(0)\right|_{2, \Omega}^{2}+\mathcal{E}(0)+\left|e_{\alpha}^{h}(0)\right|_{1, \Omega}^{2}+\left|\gamma_{\alpha \beta}(0)-\tilde{\gamma}_{\alpha \beta}^{h}(0)\right|_{0, \Omega}^{2}+\left|u_{3}(0)-\tilde{u}_{3}^{h}(0)\right|_{2, \Omega}^{2}\right. \\
& \left.+C h^{2(r-2)}\left|u_{3}(0)\right|_{r, \Omega}^{2}\right]
\end{aligned}
$$

Additionally, using the definition of $\mathcal{E}(t)$ in $(2.8)$, we get

$$
\begin{aligned}
\mathcal{E}(0) \leq & C\left[\left|\left(\dot{u}_{\alpha}^{h}-\dot{\tilde{u}}_{\alpha}^{h}\right)(0)\right|_{0, \Omega}^{2}+\left|\left(\dot{u}_{3}^{h}-\dot{\tilde{u}}_{3}^{h}\right)(0)\right|_{0, \Omega}^{2}+\gamma\left|\left(\dot{u}_{3}^{h}-\dot{\tilde{u}}_{3}^{h}\right)(0)\right|_{1, \Omega}^{2}+\left|\left(\gamma_{\alpha \beta}^{h}-\tilde{\gamma}_{\alpha \beta}^{h}\right)(0)\right|_{0, \Omega}^{2}\right. \\
& \left.+\left|\left(u_{3}^{h}-\tilde{u}_{3}^{h}\right)(0)\right|_{2, \Omega}^{2}\right] .
\end{aligned}
$$


We also make use of (3.25) and (1.4) to obtain

$$
\begin{aligned}
\left|\gamma_{\alpha \beta}(0)-\tilde{\gamma}_{\alpha \beta}^{h}(0)\right|_{0, \Omega}^{2} \leq & C\left|\left(u_{\alpha}-\tilde{u}_{\alpha}^{h}\right)(0)\right|_{1, \Omega}+C\left|\left(u_{3, \alpha}-\tilde{u}_{3, \alpha}^{h}\right)(0) u_{3, \beta}(0)\right|_{0, \Omega} \\
& +C\left|\tilde{u}_{3, \alpha}^{h}(0)\left(u_{3, \beta}-\tilde{u}_{3, \beta}^{h}\right)(0)\right|_{0, \Omega}+C\left|\left(u_{3}-\tilde{u}_{3}^{h}\right)(0)\right|_{0, \Omega} \\
\leq & C h^{s-1}\left|u_{\alpha}(0)\right|_{s, \Omega}+C\left|\left(u_{3, \alpha}-\tilde{u}_{3, \alpha}^{h}\right)(0)\right|_{1, \Omega}\left|u_{3, \beta}(0)\right|_{\epsilon, \Omega} \\
& +C\left|\left(u_{3, \beta}-\tilde{u}_{3, \beta}^{h}\right)(0)\right|_{1, \Omega}\left|\tilde{u}_{3, \alpha}^{h}(0)\right|_{\epsilon, \Omega}+C h^{r-1}\left|u_{3}(0)\right|_{r-1, \Omega} \\
\leq & C h^{s-1}\left|u_{\alpha}(0)\right|_{s, \Omega}+C h^{r-2}\left|u_{3}(0)\right|_{r, \Omega}\left[\left|u_{3}(0)\right|_{2, \Omega}+\left|\tilde{u}_{3}(0)\right|_{2, \Omega}\right]+C h^{r-1}\left|u_{3}(0)\right|_{r-1, \Omega} \\
\leq & C\left(E_{\gamma}(0)\right)\left[h^{s-1}\left|u_{\alpha}(0)\right|_{s, \Omega}+h^{r-2}\left|u_{3}(0)\right|_{r, \Omega}\right] .
\end{aligned}
$$

Hence, by applying $(3.54,3.55)$ to $(3.53)$ and recalling the definition $\mathbf{e}^{h}=\mathbf{u}^{h}-\tilde{\mathbf{u}}^{h}$, we obtain

$$
\begin{aligned}
Y_{0} \leq C\left(E_{\gamma}(0),|(\vec{p}, q)|\right)[ & \mid\left(\dot{u}_{\alpha}^{h}-\left.\dot{\tilde{u}}_{\alpha}^{h}(0)\right|_{0, \Omega} ^{2}+\left|\left(\dot{u}_{3}^{h}-\dot{\tilde{u}}_{3}^{h}\right)(0)\right|_{0, \Omega}^{2}+\gamma\left|\left(\dot{u}_{3}^{h}-\dot{\tilde{u}}_{3}^{h}\right)(0)\right|_{1, \Omega}^{2}+\left|\left(u_{3}^{h}-\tilde{u}_{3}^{h}\right)(0)\right|_{2, \Omega}^{2}\right. \\
& \left.+\left|\left(\gamma_{\alpha \beta}^{h}-\tilde{\gamma}_{\alpha \beta}^{h}\right)(0)\right|_{0, \Omega}^{2}+\left|\left(u_{\alpha}^{h}-\tilde{u}_{\alpha}^{h}\right)(0)\right|_{1, \Omega}^{2}+h^{2(s-1)}\left|u_{\alpha}(0)\right|_{s, \Omega}^{2}+h^{2(r-2)}\left|u_{3}(0)\right|_{r, \Omega}^{2}\right] .
\end{aligned}
$$

Moreover, taking $\mathbf{u}^{h}(0)=\tilde{\mathbf{u}}^{h}(0)$ we obtain

$$
\begin{aligned}
Y_{0} & \leq C\left(E_{\gamma}(0),|(\vec{p}, q)|\right)\left[h^{2(s-1)}\left|u_{\alpha}(0)\right|_{s, \Omega}^{2}+h^{2(r-2)}\left|u_{3}(0)\right|_{r, \Omega}^{2}\right] \\
& \leq C\left(E_{\gamma}(0),|(\vec{p}, q)|\right)\left[h^{2(s-1)}\left[\left|u_{\alpha}\right|_{s, 2}^{2}+\left|\dot{u}_{\alpha}\right|_{s, 2}^{2}\right]+h^{2(r-2)}\left[\left|u_{3}\right|_{r, 2}^{2}+\left|\dot{u}_{3}\right|_{r, 2}^{2}\right]\right]
\end{aligned}
$$

which is the desired inequality in (3.49).

The proof of Lemma 3.3 follows by combining the inequalities in Proposition 3.5 and 3.7.

The combination of Lemma 3.2 and Lemma 3.3 provides the proof of Theorem 1.2.

\section{REFERENCES}

[1] M. Bernadou, Méthodes d'Éléments Finis pour les Problèmes de Coques Minces. Masson, Paris-Milan-Barcelone (1994).

[2] M. Bernadou and P.G. Ciarlet, Sur l'ellipticité du modèle linéaire de coques de W.T. Koiter, in Computing Methods in Applied Sciences and Engineering (Lecture Notes in Economics and Mathematical Systems), Springer-Verlag (1976) 89-136.

[3] M. Bernadou and B. Lalanne, On the approximations of free vibration modes of a general thin shell, application to turbine blades, in The Third European Conference on Mathematics in Industry, J. Manley et al. Eds., Kluwer Academic Publishers and B.G. Teubner Stuttgart (1990) 257-264.

[4] M. Bernadou, P.G. Ciarlet and B. Miara, Existence theorems for two-dimensional linear shell theories. Technical Report 1771, Unité de Recherche INRIA-Rocquencourt (1992).

[5] M. Bernadou and J.T. Oden, An existence theorem for a class of nonlinear shallow shell problems. J. Math. Pures Appl. 60 (1981) 285-308.

[6] P.G. Ciarlet, The Finite Element Method For Elliptic Problems. North-Holland Publishing Company, Amsterdam, New York, Oxford (1978).

[7] M.C. Delfour and J.P. Zolésio, Tangential differential equations for dynamical thin/shallow shells. J. Differential Equations 128 (1995) 125-167.

[8] W. Flügge, Tensor Analysis and Continuum Mechanics. Springer-Verlag (1972).

[9] R. Glowinski, Numerical Methods for Nonlinear Variational Problems. Springer Verlag, New York (1984).

[10] R. Glowinski and O. Pironneau, Numerical methods for the first biharmonic equation and for the two-dimensional Stokes problem. SIAM Rev. 21 (1979) 167-212.

[11] R. Glowinski and M. Wheeler, Domain decomposition and mixed finite element methods for elliptic problems, in Domain Decomposition Methods for Partial Differential Equations, SIAM (1988) 144-172.

[12] A.E. Green and W. Zerna, Theoretical Elasticity. Oxford University Press, 2nd. edn. (1968).

[13] W.T. Koiter, On the nonlinear theory of thin elastic shells, in Proc. Kon. Ned. Akad. Wetensch., Vol. B (1966) 1-54.

[14] J.E. Lagnese, Boundary Stabilization of Thin Plates. SIAM, Philadelphia, Pennsylvania (1989).

[15] I. Lasiecka, Uniform stabilization of a full von Karman system with nonlinear boundary feedback. SIAM J. Control 36 (1998) $1376-1422$. 
[16] I. Lasiecka, Weak, classical and intermediate solutions to full von Karman system of dynamic nonlinear elasticity. Applicable Anal. 68 (1998) 123-145.

[17] J.L. Lions, Quelques Méthodes de Résolution des Problèmes aux Limites Non Linéaires. Dunod, Paris (1969).

[18] J.L. Lions and E. Magenes, Non-Homogeneous Boundary Value Problems and Applications, Vol. 1. Springer Verlag (1972).

[19] L. Mansfield, Analysis of finite element methods for the nonlinear dynamic analysis of shells. Numerische Mathematik 42 (1983) 213-235.

[20] R. Marchand, Finite element approximations of control problems arising in nonlinear shell theory. Ph.D. thesis, University of Virginia (1996).

[21] V.G. Mazya and T.V. Shaposhnikova, Theory of Multipliers in Spaces of Differentiable Functions. Pitman (1985).

[22] A. Raoult, Analyse mathématique de quelques modèles de plaques et de poutres élastiques ou élasto-plastiques. Doctoral Dissertation, Université Pierre et Marie Curie, Paris (1988).

[23] H.L. Royden, Real Analysis. Macmillan Publishing Company, 3rd edn. (1988).

[24] V.I. Sedenko, The uniqueness of generalized solutions of initial boundary value problem for Marguerre-Vlasov equation in the nonlinear oscillation theory of shallow shells. Izwestia Vysshyh Uchebnych Zavedenij (1994) 1-2.

[25] V. Thomee, Galerkin Finite Element Methods for Parabolic Problems. Springer Verlag (1984). 